\title{
DISEÑO SIMPLIFICADO POR TORSIÓN SÍSMICA ESTÁTICA
}

\author{
José Alberto Escobar $S^{(1)}$, Antonio Mendoza $S .{ }^{(1)}$ y Roberto Gómez M. ${ }^{(1)}$
}

\begin{abstract}
RESUMEN
Se propone un método denominado Procedimiento Simplificado de Diseño, PSD, que reduce los cálculos en el diseño por torsión sísmica estática de edificios. Para ello se estudia la distribución de las fuerzas cortantes entre los elementos resistentes de entrepiso en edificios diseñados por torsión sísmica y se analizan los factores que afectan la excentricidad de diseño, particularmente la excentricidad accidental. Se demuestra que el efecto de la torsión debido a esta excentricidad siempre es aditivo en los elementos estructurales. Con el PSD propuesto es suficiente un solo análisis de la estructura tridimensional, en cada una de sus direcciones ortogonales, para calcular las fuerzas de diseño en los elementos estructurales. El procedimiento propuesto se aplica a un ejemplo en el que se obtienen los mismos resultados que con el método tradicional.
\end{abstract}

\section{SUMMARY}

A new procedure called $P S D$ to simplify the current static seismic torsion design analysis for buildings is proposed. In order to do it, the distribution of the shear forces among the story resistant elements designed by torsion, and the factors whose affect the design eccentricity, especially the accidental eccentricity, are studied. It is demonstrated that the effect of the accidental eccentricity is always additive for the structural elements. By using the proposed PSD just one analysis in each one of the orthogonal directions of the three-dimensional model of the structure is enough to determine the design forces of the structural elements. An example applying the proposed procedure is studied. The results are the same as those obtained using the traditional design procedure.

\section{INTRODUCCIÓN}

De acuerdo con la filosofía de diseño sísmico actual, cuando las estructuras son sometidas a sismos intensos, deberán ser capaces de resistir deformaciones inelásticas significativas conservando su capacidad de carga. En el caso de edificios asimétricos estas deformaciones inelásticas provienen fundamentalmente de la traslación y rotación de sus entrepisos. Desde este punto de vista, el comportamiento de estas estructuras debe ser tal que, además de no exceder los límites establecidos para los desplazamientos laterales, tienen que controlar los adicionales debidos a torsión dentro de los límites permisibles de desplazamiento de entrepiso.

Artículo recibido el 17 de septiembre de 2002 y aprobado para su publicación el 15 de octubre de 2003. Se aceptarán comentarios y/o discusiones hasta cinco meses después de su publicación.

(1) Instituto de Ingeniería, UNAM, Ciudad Universitaria, Apdo. Postal 7740, Coyoacan 04510, México, DF., México jess@pumas.iingen.unam.mx, rgom@pumas.iingen.unam.mx 
Considerando los efectos anteriores, en los reglamentos actuales se permite analizar las estructuras de edificios tomando en cuenta únicamente traslación de los entrepisos. La torsión sísmica es incluida al distribuir la fuerza cortante producida por este efecto entre los elementos resistentes al considerar un momento de torsión en cada entrepiso, obtenido del producto de la fuerza cortante sísmica en cada dirección de análisis y dos valores de la excentricidad de diseño.

Al igual que otros reglamentos de diseño por torsión, el actual Reglamento de Construcciones para el Distrito Federal, RCDF, a través de sus Normas Técnicas Complementarias para Diseño por Sismo, NTCDS (NTC-1995), permite llevar a cabo un análisis estático de las estructuras de edificios, considerando que las fuerzas sísmicas actúan en el centro de masas de cada uno de sus pisos, y que los momentos de torsión correspondientes se distribuyen entre los elementos resistentes. Sin embargo, las solicitaciones que se obtienen de este análisis son diferentes a las que resultan de un análisis dinámico tridimensional, en donde se considera el acoplamiento entre vibraciones torsionales y traslacionales. En general, este acoplamiento puede ocasionar una amplificación dinámica de los momentos de torsión. En estudios de estructuras con comportamiento elástico este efecto es muy claro, principalmente cuando la relación entre frecuencias desacopladas es cercana a la unidad (Elorduy y Rosenblueth, 1968). Para tomar en cuenta este efecto, se utiliza un factor de amplificación de la excentricidad estática que incrementa las fuerzas de diseño mediante un momento de diseño cuyo valor es mayor que el calculado estáticamente.

Por otro lado, los momentos de torsión en edificios reales difieren de los obtenidos en un análisis dinámico debido a factores no considerados explícitamente (Rosenblueth, 1979). Por una parte se pueden atribuir a las torsiones inducidas por el componente rotacional del terreno y a la diferencia en la llegada de las ondas sísmicas a los apoyos de las estructuras, y por la otra, a la diferencia entre las propiedades reales y las calculadas. Este aspecto está asociado a las incertidumbres inherentes a las propiedades estructurales, ya que aun las estructuras nominalmente simétricas pueden ser afectadas por torsión (Pekau y Guimond, 1988; De la Llera y Chopra, 1994a, 1994b; Escobar, 1994, 1996; Escobar y Ayala, 1998). En la actualidad, resulta prácticamente imposible estimar con precisión estas torsiones denominadas accidentales. La manera usual de considerarlas en el diseño sísmico de estructuras, es incluyendo un momento de torsión adicional, que se obtiene de suponer que la fuerza cortante que actúa en el entrepiso se desplaza de su posición original. A este desplazamiento de la fuerza cortante se le denomina excentricidad accidental. Comúnmente, la excentricidad accidental se expresa como un porcentaje de la dimensión máxima de la planta de la estructura que es perpendicular a la dirección del sismo.

En el presente trabajo se analizan los factores que afectan la excentricidad de diseño, particularmente la excentricidad accidental, con el objetivo de simplificar su manejo en el criterio de diseño por torsión sísmica estática del RCDF vigente. Se demuestra que, de acuerdo con el $\mathrm{RCDF}$, las fuerzas producidas por esta excentricidad, siempre son aditivas en los elementos estructurales. Con base en esta observación se propone un procedimiento que simplifica el diseño por torsión sísmica estática. 


\section{REGLAMENTO DE CONSTRUCCIONES PARA EL DF}

De acuerdo con el RCDF, para calcular las fuerzas de diseño de los elementos resistentes de cada entrepiso primero se distribuye la fuerza cortante total $V_{j}$ del mismo de acuerdo con la rigidez lateral, $k_{i}$, de cada elemento, obteniéndose un cortante directo de diseño $V_{d i}$, esto es, para cada una de las direcciones ortogonales de la estructura:

$$
V_{d i}=V_{j} \frac{k_{i}}{\sum k_{i}}
$$

La fuerza cortante debida al efecto de la torsión sísmica se obtiene del momento torsionante originado por la aplicación de la fuerza cortante de entrepiso y una excentricidad de diseño $e_{d}$ que puede adoptar uno de dos valores. La mayoría de los reglamentos de diseño sísmico especifican como excentricidad de diseño expresiones con el siguiente formato:

$e_{d}=\alpha e_{s}+\beta b$

o:

$e_{d}=\delta e_{s}-\beta b$

donde $\alpha$ y $\delta$ son factores de amplificación dinámica, $e_{s}$ es la excentricidad estructural o estática (definida en la siguiente sección), $\beta$ es el factor de excentricidad accidental, y $b$ es la dimensión máxima en planta de la estructura, perpendicular a la dirección de la excitación sísmica que se analiza. De las ecuaciones (2) se utiliza como excentricidad de diseño la que ocasione los efectos más desfavorables en cada uno de los elementos resistentes.

Las fuerzas cortantes de diseño debidas a la torsión del entrepiso, $V_{t i}$, se obtienen de distribuir del momento torsionante de acuerdo con la contribución de los elementos estructurales a la rigidez torsional del mismo, esto es:

$V_{t i}=\frac{k_{i} x_{i}}{K_{\theta}} V_{j} e d$

donde $K_{\theta}=\sum x_{i}^{2} k_{i}+\sum y_{i}^{2} k_{i} ; x_{i}, y_{i}$ son las coordenadas del $i$-ésimo elemento estructural, con respecto al centro de rigidez o de torsión en las direcciones ortogonales $X$, $Y$ respectivamente, y $k_{i}$ es la rigidez de los elementos en cada una de las direcciones ortogonales del sistema estructural.

Considerando los efectos anteriores, es posible analizar las estructuras de edificios tomando en cuenta únicamente la traslación de los entrepisos. Adicionalmente, en la aplicación de las ecuaciones anteriores se deberán tomar en cuenta las especificaciones de las Normas Técnicas Complementarias para Diseño por Sismo, del RCDF que han ido evolucionando con el tiempo. Así, en las NTCDS de 1987 (NTC-1987), se establecen las condiciones de regularidad que debe 
cumplir un edificio para considerar el fenómeno de torsión. Si alguna de estas condiciones no se cumple, el factor de comportamiento sísmico para diseñar la estructura se deberá multiplicar por 0.8 para aumentar la resistencia de los elementos estructurales. También se introduce el concepto de centroide de las resistencias de los elementos estructurales, el cual no tiene una definición precisa y no se vuelve a mencionar en las ediciones posteriores de las NTCDS.

En 1995, en las especificaciones para diseño por torsión de las NTCDS (NTC-1995), además de tenerse que cumplir con las condiciones de regularidad anteriores, se propuso que en las estructuras para las que el valor del factor de comportamiento sísmico fuera igual o mayor que 3 , en ninguno de sus entrepisos la excentricidad estática calculada deberá exceder de $0.2 b$. También se establece que el valor de la excentricidad de diseño en cada una de las direcciones ortogonales de análisis de la estructura, no será menor que la mitad del valor máximo de la excentricidad estática para los entrepisos que se hallan abajo del que se considera, ni se tomará el momento por torsión de ese entrepiso menor que la mitad del máximo calculado para los entrepisos que están arriba del considerado. De acuerdo con estas especificaciones, en las ecuaciones (2), $\alpha=1.5, \delta=1.0$ y $\beta=0.1$ (NTC-1995).

Las modificaciones más recientes para el diseño de estructuras por torsión fueron realizadas en la propuesta de las NTCDS de 2001 (NTC-2001). En ellas se indica que los elementos estructurales deben conservar, por lo menos, la resistencia que se calculó para soportar el cortante directo. También, las estructuras irregulares se clasifican en irregular con una sola condición no cumplida, irregular con más de dos condiciones no cumplidas y fuertemente irregular que se genera cuando se tiene una excentricidad mayor que $0.2 b$ en cualquier entrepiso. En estos casos, los valores de los coeficientes que afectan al factor de comportamiento sísmico son $0.9,0.8$ y 0.7 , respectivamente.

Adicionalmente, se deberán tener en cuenta los efectos bidireccionales del sismo, especificados en todas las versiones de las NTCDS.

\section{EDIFICIOS DE VARIOS PISOS}

Lo hasta aquí comentado es directamente aplicable a edificios de un piso. Sin embargo, en el diseño por torsión sísmica estática de edificios de varios pisos existen dos definiciones de excentricidad estática para calcular el momento torsionante (Cheung y Tso, 1986; Tso, 1990) que a continuación se describen.

\section{Excentricidad de piso}

Es la distancia entre el centro de masas, $C M$, y el centro de torsión, $C T$ correspondiente para cada una de las direcciones $X$ e $Y$ de la estructura, esto es

$$
\begin{aligned}
& e_{s}=x_{C M}-x_{C T} \\
& e_{s}=y_{C M}-y_{C T}
\end{aligned}
$$


Las coordenadas $x_{C M}, y_{C M}$ del $C M$ del j-ésimo piso se calculan como

$x_{C M}=\frac{\Sigma P_{i} y_{i}}{\Sigma P_{i}}$

$y_{C M}=\frac{\Sigma P_{i} x_{i}}{\Sigma P_{i}}$

donde $P_{i}$ son las cargas verticales en el piso, y $x_{i}$ y $y_{i}$ son sus coordenadas respecto a un punto de referencia.

Para calcular las coordenadas $x_{C T}, y_{C T}$ del $C T$ del piso, se pueden utilizar los cortantes directos. Estos pueden obtenerse al aplicar las fuerzas sísmicas laterales $F x_{j}$ y $F y_{j}$, calculadas con un análisis sísmico estático, en los $C M$ correspondientes, permitiendo únicamente la traslación pura de los pisos. Así, las coordenadas del $C T$ se calculan con las ecuaciones siguientes

$$
\begin{aligned}
& x_{C T}=\frac{\sum\left(V_{d y i, j}-V_{d y i, j-1}\right) x_{i}}{F y_{j}} \\
& y_{C T}=\frac{\sum\left(V_{d x i, j}-V_{d x i, j-1}\right) y_{i}}{F x_{j}}
\end{aligned}
$$

donde $V_{d x i, j}$ y $V_{d y i, j}$ son los cortantes directos del $i$-ésimo elemento resistente; y $x_{i}$, $y_{i}$ son sus coordenadas respecto a un punto de referencia en las direcciones $X$ e $Y$ en el entrepiso $j$, respectivamente.

El momento de torsión del $j$-ésimo piso para cada una de las direcciones $X$ e $Y$ de la estructura será

$$
\begin{aligned}
& T_{j}=F x_{j} e_{s} \\
& T_{j}=F y_{j} e_{s}
\end{aligned}
$$

El momento de torsión de entrepiso se obtiene sumando los momentos torsionantes de todos los pisos que se encuentran sobre éste. Así, el momento torsionante del $j$-ésimo entrepiso, para cada una de las direcciones $X$ e $Y$ está dado por

$$
M_{j}=\sum_{m=j}^{n} T_{m}
$$

donde $n$ es el número de entrepisos. 


\section{Excentricidad de entrepiso}

Es la distancia entre el centro de cortantes, $C C$, y el centro de rigideces, $C R$, de cada una de las direcciones $X$ e $Y$ de los entrepisos esto es

$$
\begin{aligned}
& e_{s}=x_{C C}-x_{C R} \\
& e_{s}=y_{C C}-y_{C R}
\end{aligned}
$$

Las coordenadas $x_{C C}$ y $y_{C C}$ del $C C$ del j-ésimo entrepiso se calculan como

$$
\begin{aligned}
x_{C C} & =\frac{\sum F y_{j} x_{C M}}{V y_{j}} \\
y_{C C} & =\frac{\sum F x_{j} y_{C M}}{V x_{j}}
\end{aligned}
$$

donde $F_{x j}$ y $F_{y j}$, son las fuerzas sísmicas laterales aplicadas en $C M ; V x_{j}$ y $V y_{j}$ son los cortantes del entrepiso $j$ en las direcciones $X$ e $Y$, respectivamente.

sigue

Las coordenadas $x_{C R}, y_{C R}$ del $C R$ se pueden calcular utilizando los cortantes directos como

$$
\begin{aligned}
& x_{C R}=\frac{\Sigma\left(V_{d y i} x_{i}\right)}{V y_{j}} \\
& y_{C R}=\frac{\Sigma\left(V_{d x i} y_{i}\right)}{V x_{j}}
\end{aligned}
$$

o mediante las rigideces de los elementos resistentes como

$$
\begin{aligned}
& x_{C R}=\frac{\sum\left(k_{i} x_{i}\right)}{\sum k_{i}} \\
& y_{C R}=\frac{\sum\left(k_{i} y_{i}\right)}{\sum k_{i}}
\end{aligned}
$$

Con esta definición, el momento de torsión de entrepiso se obtiene directamente como el producto de la fuerza cortante y la excentricidad de entrepiso. Así, el momento torsionante del $j$ ésimo entrepiso para cada una de las direcciones ortogonales $X$ e $Y$ será 
$M_{j}=V_{j} e_{s}$

Utilizando los conceptos de excentricidad de piso y de entrepiso descritos, en las tablas 1 y 2 se presentan los resultados de los momentos torsionantes calculados para la dirección $X$ de los entrepisos del edifico estudiado por Bazán y Meli (1998), mostrado en la fig 1. Se puede observar que, como era de esperarse, los valores del momento de torsión calculados con las dos definiciones de excentricidad estática son prácticamente iguales entre sí. En el Apéndice A se presenta el cálculo de los $C T$ y $C R$ de piso y de entrepiso para la dirección $X$ del mismo edificio.

Tabla 1. Momentos de torsión calculados con la excentricidad de piso. Dirección $X$.

\begin{tabular}{|c|c|c|c|c|c|c|}
\hline Piso & $F x_{i}(\mathrm{t})$ & $\begin{array}{c}y_{C M}(\mathrm{~m}) \\
\mathrm{ec}(5)\end{array}$ & $\begin{array}{c}y_{C T}(\mathrm{~m}) \\
\mathrm{ec}(6)\end{array}$ & $\begin{array}{c}e_{s}(\mathrm{~m}) \\
\mathrm{ec}(4)\end{array}$ & $\begin{array}{c}T_{j}(\mathrm{t}-\mathrm{m}) \\
\mathrm{ec}(7)\end{array}$ & $\begin{array}{c}M_{j}(\mathrm{t}-\mathrm{m}) \\
\mathrm{Ec}(8)\end{array}$ \\
\cline { 1 - 6 } Entrepiso & 23.77 & 3.75 & 4.73 & -0.98 & -23.29 & \\
\hline 5 & & & & & & -23.29 \\
\hline 5 & 25.75 & 5.50 & 7.17 & -1.67 & -43.0 & \\
\hline 4 & & & & & & -66.29 \\
\hline 3 & 24.76 & 5.50 & 5.47 & 0.03 & 0.74 & \\
\hline 3 & & & & & & -65.55 \\
\hline 2 & 17.33 & 5.50 & 5.82 & -0.32 & -5.55 & \\
\hline 2 & & & & & & -71.09 \\
\hline 1 & 11.89 & 6.30 & 5.82 & 0.48 & 5.71 & \\
\hline 1 & & & & & & -65.39 \\
\hline
\end{tabular}

Tabla 2. Momentos de torsión calculados con la excentricidad de entrepiso. Dirección $X$.

\begin{tabular}{|c|c|c|c|c|c|}
\hline Entrepiso & $V x_{j}(\mathrm{t})$ & $\begin{array}{c}y_{C C}(\mathrm{~m}) \\
\mathrm{ec}(10)\end{array}$ & $\begin{array}{c}y_{C R}(\mathrm{~m}) \\
\mathrm{ec}(11 \mathrm{o} 12)\end{array}$ & $\begin{array}{c}e_{s}(\mathrm{~m}) \\
\mathrm{ec}(9)\end{array}$ & $\begin{array}{c}M_{j}(\mathrm{t}-\mathrm{m}) \\
\mathrm{Ec}(13)\end{array}$ \\
\hline 5 & 23.77 & 3.75 & 4.73 & -0.98 & -23.29 \\
\hline 4 & 49.52 & 4.66 & 6.00 & -1.34 & -66.36 \\
\hline 3 & 74.28 & 4.94 & 5.82 & -0.88 & -65.37 \\
\hline 2 & 91.61 & 5.05 & 5.82 & -0.77 & -70.54 \\
\hline 1 & 103.50 & 5.19 & 5.82 & -0.63 & -65.21 \\
\hline
\end{tabular}

\section{DISTRIBUCIÓN DEL CORTANTE POR TORSIÓN ENTRE LOS ELEMENTOS RESISTENTES}

Para simplificar el procedimiento de diseño por torsión sísmica estática actual, se analizan las ecuaciones en las que éste se basa. Así, de acuerdo con las ecuaciones (1) y (3), el diseño por torsión del $i$-ésimo elemento resistente de un entrepiso debe incluir un cortante directo $V_{d i} \mathrm{y}$ un cortante por torsión $V_{t i}$, esto es

$V_{i}=V_{d i} \pm V_{t i}$ 
En la fig 2 se presenta esquemáticamente el efecto del cortante directo y el de torsión en los elementos resistentes de un sistema estructural simple. Sustituyendo las ecuaciones (1) y (3) en la ecuación (14) se obtiene

$V_{i}=\frac{k_{i}}{\Sigma k_{i}} V_{j} \pm \frac{k_{i} x_{i}}{K_{\theta}} V_{j} e$

donde $x_{i}$ es la distancia del $i$-ésimo elemento estructural al $C R$ o al $C T$.

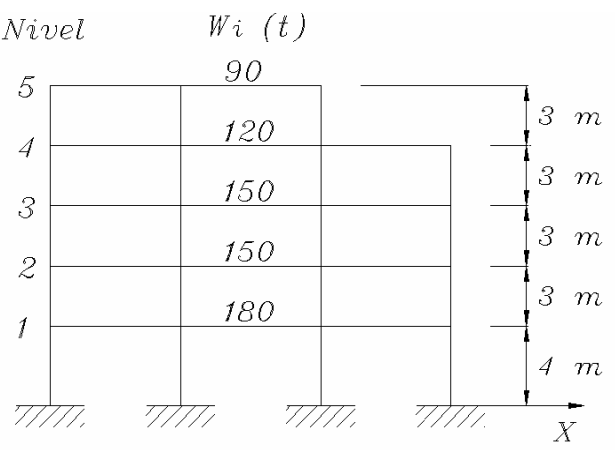

a) Elevación

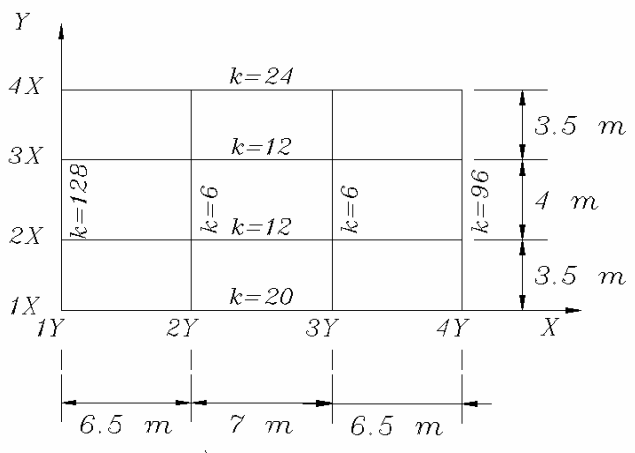

b) Entrepiso 1 a 3

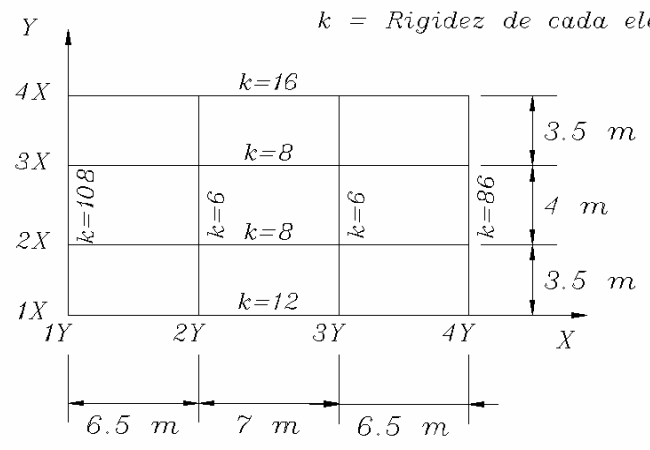

c) Entrepiso 4

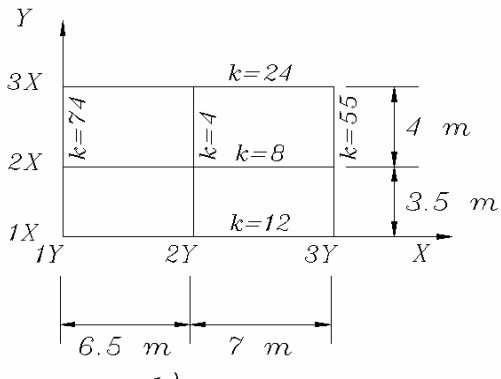

d) Entrepiso 5

Figura 1. Edificio de cinco pisos estudiado (Bazán y Meli, 1998).

Como se puede observar en la ecuación (15), uno de los parámetros que interviene de manera fundamental en la distribución de las fuerzas por torsión en edificios, es la rigidez torsional de entrepiso $K_{\theta}$. Con base en este concepto, Tso y Wong (1993) han sugerido un parámetro estructural denominado radio de giro normalizado, $\rho$, para estudiar el comportamiento de estructuras. Este parámetro también permite establecer valores mínimos de la rigidez a torsión de una estructura para mantener sus desplazamientos relativos de entrepiso dentro de límites de comportamiento aceptables. En el caso de comportamiento no lineal, este parámetro es útil para controlar las demandas de ductilidad excesivas que pudieran presentarse en los elementos resistentes (Escobar, et al., 1999; Padilla, 2002). Así, el radio de giro normalizado para cada una de las dos direcciones ortogonales de un entrepiso de un sistema estructural se define como 


$$
\rho=\frac{1}{b} \sqrt{\frac{K_{\theta}}{\sum k_{i}}}
$$

La relación de este parámetro con la de frecuencias torsional y lateral desacopladas, $\Omega$, está dada como

$$
\rho=\frac{1}{b} \sqrt{\frac{\Omega m_{\theta}}{m}}
$$

donde $m$ y $m_{\theta}$ son la masa traslacional y rotacional del entrepiso, respectivamente.
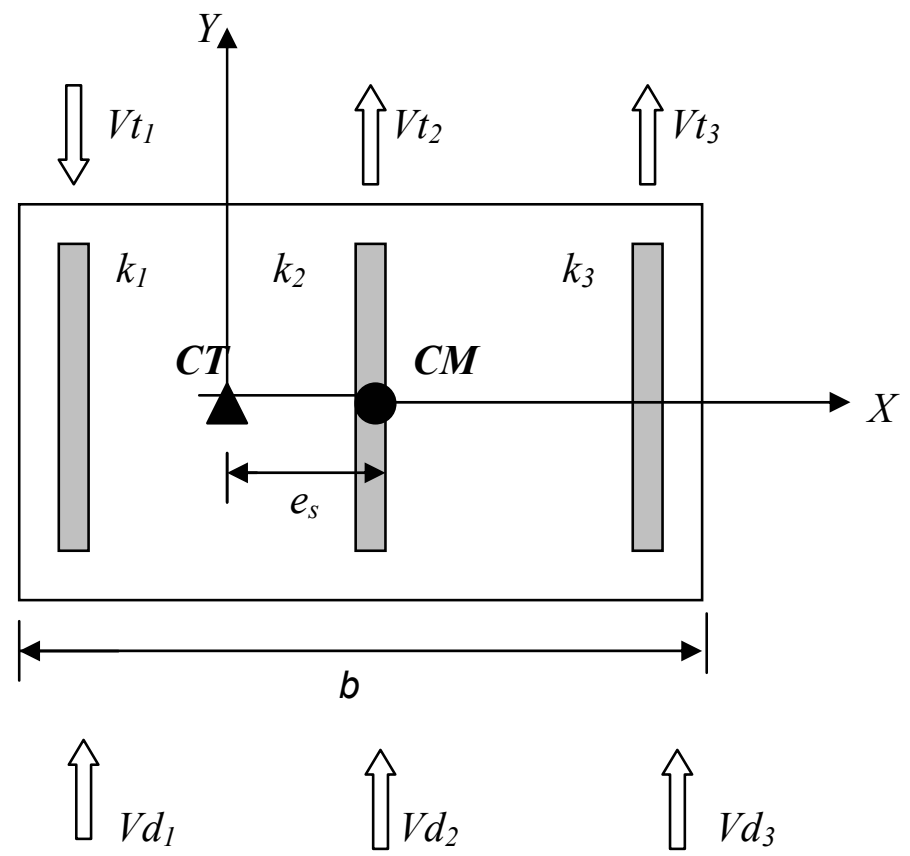

Figura 2. Distribución del cortante directo y del cortante por torsión entre los elementos resistentes en dirección $Y$.

Las ecuaciones (1) a (3) permiten llevar a cabo el diseño por torsión, mientras que la ecuación (16) se puede considerar como un requisito adicional que se debe cumplir para garantizar que el comportamiento no lineal de la estructura esté dentro de un límite de comportamiento adecuado (Escobar et al., 1999; Padilla, 2002). De la ecuación (16) se tiene que

$$
K_{\theta}=\Sigma k_{i} \rho^{2} b^{2}
$$

Entonces, sustituyendo la ecuación (17) en la (15) y simplificando se obtiene 


$$
V_{i}=V_{d i}\left(1 \pm \frac{e}{\rho^{2} b^{2}} x_{i}\right)
$$

Sustituyendo las ecuaciones (2) en la expresión anterior, se tiene que para los elementos localizados del mismo lado del $C M$ o del $C C$ con respecto al $C T$ o al $C R$, denominados elementos flexibles, la resistencia estará dada por el efecto más desfavorable calculado con las siguientes expresiones (Fig. 2)

$$
V_{i}=V_{d i}\left(1+\frac{\alpha e_{s}+\beta b}{\rho^{2} b^{2}} x_{i}\right)=V_{d i}\left(1+\frac{\beta b}{\rho^{2} b^{2}} x_{i}+\frac{\alpha e_{s}}{\rho^{2} b^{2}} x_{i}\right)
$$

O

$$
V_{i}=V_{d i}\left(1+\frac{\delta e_{s}-\beta b}{\rho^{2} b^{2}} x_{i}\right)=V_{d i}\left(1-\frac{\beta b}{\rho^{2} b^{2}} x_{i}+\frac{\delta e_{s}}{\rho^{2} b^{2}} x_{i}\right)
$$

Comparando la ecuación (19) con la (20) se obtiene que

$$
\left.\beta b+\alpha e_{S}\right\rangle-\beta b+\delta e_{S}
$$

Entonces, el diseño de los elementos flexibles lo rige la ecuación (19).

Por otro lado, la resistencia los elementos localizados del mismo lado del $C T$ o del $C R$ con respecto al $C C$ o al $C M$, denominados elementos rígidos, se obtendrá calculando el efecto más desfavorable con las siguientes expresiones

$$
V_{i}=V_{d i}\left(1-\frac{\alpha e_{s}+\beta b}{\rho^{2} b^{2}} x_{i}\right)=V_{d i}\left(1-\frac{\beta b}{\rho^{2} b^{2}} x_{i}-\frac{\alpha e_{s}}{\rho^{2} b^{2}} x_{i}\right)
$$

y

$$
V_{i}=V_{d i}\left(1-\frac{\delta e_{s}-\beta b}{\rho^{2} b^{2}} x_{i}\right)=V_{d i}\left(1+\frac{\beta b}{\rho^{2} b^{2}} x_{i}-\frac{\delta e_{s}}{\rho^{2} b^{2}} x_{i}\right)
$$

Comparando la ecuación (22) con la (23) se tiene que

$$
-\beta b-\alpha e_{S}<\beta b-\delta e_{S}
$$

En este caso la ecuación (23) rige el diseño de los elementos rígidos. 


\section{CORTANTE DEBIDO A LA TORSIÓN POR EXCENTRICIDAD ACCIDENTAL}

En el RCDF, el valor de la excentricidad accidental corresponde al 10\% de la dimensión máxima de la planta de la estructura que es perpendicular a la dirección de aplicación del sismo, esto es, $\beta=0.1$. Por otro lado, el RCDF vigente define como excentricidad torsional de rigideces de cada entrepiso, a "la distancia entre el centro de torsión del entrepiso correspondiente y la fuerza cortante en dicho entrepiso". Así, los efectos de la torsión accidental se incluyen mediante un momento torsionante que se obtiene de suponer que la fuerza cortante que actúa en cada entrepiso se desplaza una cantidad $\pm \beta$ b de su posición original, tal como se indica en las ecuaciones (2) para calcular la excentricidad de diseño. Entonces, para incluir los efectos de la torsión accidental es necesario hacer un análisis de la estructura para cada posición del $C C$ desplazado de su posición nominal esta cantidad.

Es importante mencionar que, debido a las limitaciones que existían en el análisis estructural de modelos más elaborados, este procedimiento fue desarrollado para aplicarlo en el cálculo de marcos planos. Su uso en modelos tridimensionales de estructuras conduce a tener que realizar un número de análisis igual a $4^{n p}$ donde $n p$ es el número de pisos.

A continuación se analizan las ecuaciones que establece el RCDF para diseño por torsión sísmica estática con el objetivo de desarrollar un procedimiento para incluir estos efectos de manera más simple que como se hace actualmente.

En las ecuaciones (19) y (23) que rigen el diseño de los elementos resistentes se aprecia que el efecto de la torsión debido a la excentricidad accidental siempre es aditivo. Esto implica que la torsión ocasionada por la excentricidad accidental se traduce en un incremento de la resistencia lateral total de las estructuras. Esta observación coincide con las hechas anteriormente por Tso y Wong (1993), Escobar (1994) y Paulay (1997). En las ecuaciones mencionadas, el efecto de la excentricidad accidental para el $i$-ésimo elemento estructural, tanto rígido como flexible, se puede representar mediante un Factor de Excentricidad Accidental, FEA, que para el $i$-ésimo elemento estructural está dado como

$$
F E A_{i}=\frac{\beta}{\rho^{2} b} x_{i}
$$

Por otro lado, el valor de la distancia $x_{i}$ en la ecuación (25), se puede representar adimensionalmente como un porcentaje $\zeta_{i}$ de la dimensión $b$ de la planta del entrepiso, esto es

$$
x_{i}=\zeta_{i} b
$$

Sustituyendo la ecuación anterior en la ecuación (25)

$$
F E A_{i}=\frac{\beta}{\rho^{2}} \zeta_{i}
$$


En la fig 3, se presenta la variación del FEAi con respecto a $\rho^{2}$, para $\beta=0.1$. Se puede observar que los valores máximos del FEAi se presentan para $\zeta_{i}=1$ (lo que implica que $x_{i}=b \mathrm{y}$ que el centro de torsión está localizado en un borde de la planta).

A partir de mediciones en una estructura real, nominalmente simétrica, De la Llera y Chopra (1994b), determinaron ecuaciones para calcular factores de excentricidad accidental y calibrar el valor del coeficiente $\beta$.

\section{CORTANTE DEBIDO A LA TORSIÓN POR EXCENTRICIDAD ESTÁTICA}

En las ecuaciones (19) y (23) se observa que el efecto de la torsión debido a excentricidad estática está en función del cortante directo, de la excentricidad estructural o estática, de los valores de los factores de amplificación dinámica y de la posición de cada elemento con respecto al $C T$ o al $C R$. Todos estos parámetros se pueden agrupar mediante un Factor de Excentricidad Estructural, FES, que se define a continuación.

De las ecuaciones (19) y (26) se tiene que el efecto de la excentricidad estática para los elementos flexibles está dado por

$$
\text { FESf }_{i}=\frac{\alpha e}{\rho^{2}} \zeta_{i}
$$

donde $e=e_{S} / b$ es la excentricidad estructural normalizada. Análogamente, de las ecuaciones (23) y (26), se tiene que el efecto de la excentricidad estática para los elementos rígidos está dado por

$$
F E S r_{i}=-\frac{\delta e}{\rho^{2}} \zeta_{i}
$$

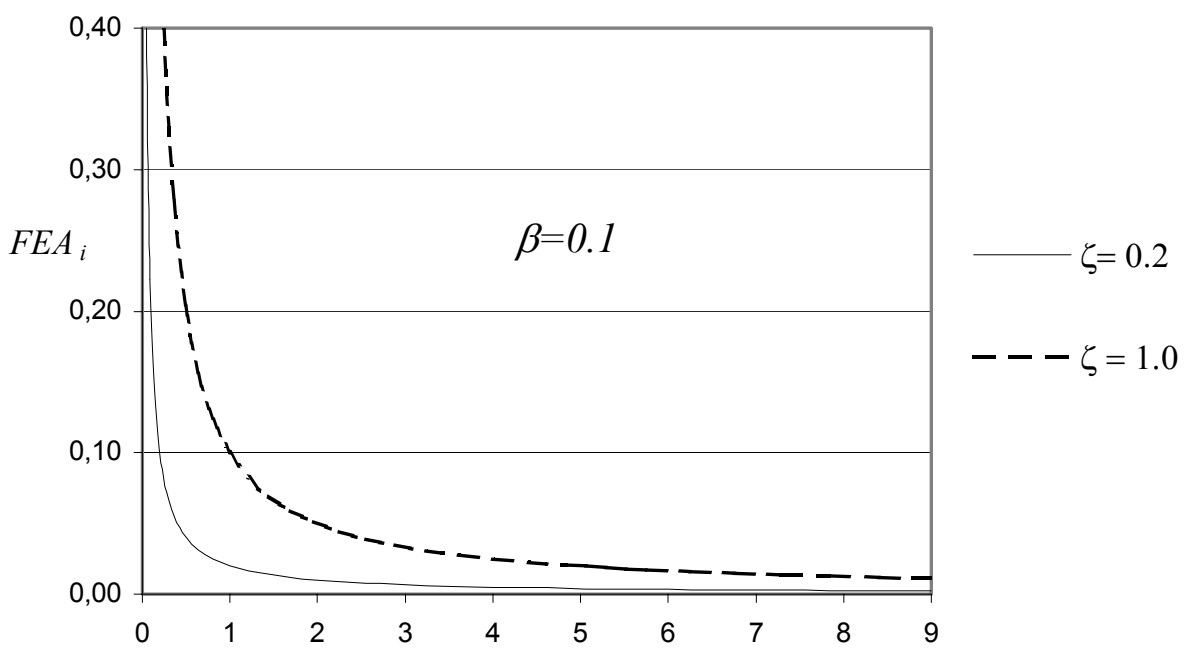

Figura 3. Valor mínimo y máximo del Factor $\rho$ ele Excentricidad Accidental, $F E A_{i}$ con respecto a $\rho^{2}$. 


\section{EFECTO DE LA TORSIÓN EN EL DISEÑO POR TORSIÓN SÍSMICA ESTÁTICA}

En general, el efecto de la torsión sísmica en el diseño por torsión sísmica estática, está en función del cortante directo, del efecto ocasionado por la excentricidad accidental y por la excentricidad estática amplificada. Estos dos últimos conceptos representan una amplificación por torsión del cortante directo para cada uno de los elementos estructurales. Por lo tanto, el efecto total debido a la torsión sísmica en éstos se puede representar mediante un Factor de Amplificación por Torsión, FAT, esto es

$$
F A T_{i}=1+F E A_{i}+F E S_{i}
$$

Con el FAT, el diseño por torsión sísmica estática se puede simplificar como se describe a continuación.

\section{Elementos flexibles}

De las ecuaciones (19), (27) y (28), se tiene que el efecto de la torsión sísmica estática en el diseño de elementos flexibles estará dado por

$$
\text { FATf }_{i}=1+\frac{\beta}{\rho^{2}} \zeta_{i}+\frac{\alpha e}{\rho^{2}} \zeta_{i}
$$

Simplificando esta ecuación se obtiene que el FAT para el $i$-ésimo elemento flexible está dado por

$$
\text { FATf }_{i}=1+\frac{\zeta_{i}}{\rho^{2}}(\beta+\alpha e)
$$

El cortante total de los elementos flexibles será

$$
V_{i}=\operatorname{FATf}_{i}\left(V_{d i}\right)
$$

\section{Elementos rígidos}

De las ecuaciones (23), (27) y (29), se tiene que el FAT para el $i$-ésimo elemento rígido está dado por

$$
F_{i T r}=1+\frac{\zeta_{i}}{\rho^{2}}(\beta-\delta e)
$$


En este caso, el cortante total será

$V_{i}=F_{i} \operatorname{Tr}_{i}\left(V_{d i}\right)$

De las ecuaciones anteriores se observa que el diseño por torsión sísmica estática se puede simplificar multiplicando el valor del cortante directo de cada uno de los elementos resistentes por su correspondiente $F A T$. De la misma forma, las fuerzas de diseño en los elementos estructurales (momentos flexionantes, fuerzas axiales cortantes, etc.), se obtienen multiplicando los efectos ocasionados por los cortantes directos, por el FAT correspondiente. Esta es la base del Procedimiento Simplificado de Diseño $P S D$ para diseño por torsión sísmica estática que aquí se propone.

Así, a diferencia del método tradicional de diseño por torsión sísmica estática, en el que, una vez que se han determinado los cortantes directos y por torsión que deberá soportar cada elemento resistente, las fuerzas de diseño se calculan mediante un análisis individual de cada uno de ellos, con el PSD es necesario hacer tan sólo un análisis de la estructura en cada una de sus dos direcciones ortogonales (los mismos que se llevan a cabo para calcular el $C T$ o el $C R$ ) para obtener los efectos de los cortantes directos y corregirlos. Además, con la clasificación que se hace de los elementos estructurales es posible tener una mejor interpretación de la distribución de los cortantes por torsión entre ellos.

\section{DISCUSIÓN SOBRE LAS ECUACIONES DE DISEÑO}

Tso y Wong, (1995) desarrollaron la representación esquemática de las ecuaciones (19) y (20); y (22) y (23) para los elementos flexibles y rígidos, respectivamente (figs 4 y 5). El cálculo del mínimo de estas dos últimas ecuaciones representa el punto donde se localiza el menor valor del incremento de la resistencia de los elementos rígidos. Así, sumando las ecuaciones (22) y (23), e igualando a cero, se obtiene

$(\alpha+\delta) \frac{e_{s}}{\rho^{2} b^{2}} x_{i}-2=0$

de donde

$e_{s}=\frac{2 \rho^{2} b^{2}}{(\alpha+\delta) x_{i}}=e_{c}$

En esta ecuación, $e_{c}$ es la excentricidad crítica, y establece el punto donde cambia la pendiente de la curva que representa la resistencia del elemento rígido (fig 5). 
Como se estableció, y de acuerdo con las figs 4 y 5 , el diseño de los elementos flexibles lo rige la ecuación (19). Sin embargo, el de los elementos rígidos lo regirá la ecuación (22) si $e_{c}<e_{s}$, o la ecuación (23) si $e_{c}>e_{s}$. De donde el FAT para estos elementos se calculará como

$$
\begin{aligned}
& \text { FATr }_{i}=1-\frac{\zeta_{i}}{\rho^{2}}(\beta+\alpha e) ; \text { si } e_{c}\left\langle e_{S}\right. \\
& \text { FATr } \left._{i}=1+\frac{\zeta_{i}}{\rho^{2}}(\beta-\delta e) ; \quad \text { si } e_{c}\right\rangle e_{S}
\end{aligned}
$$

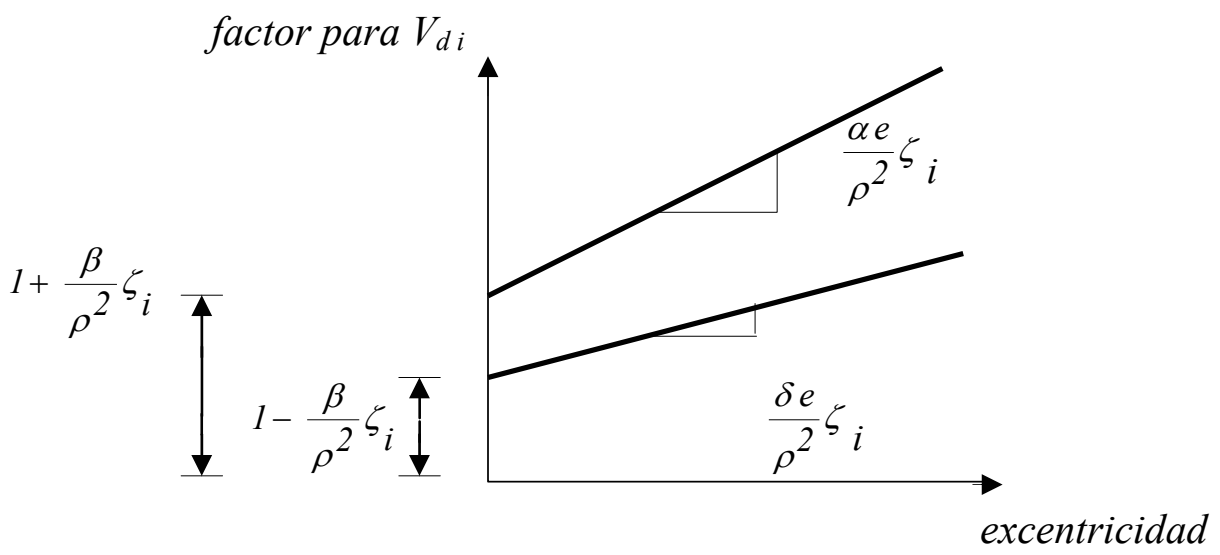

Figura 4. Representación esquemática de la variación del incremento del cortante directo con respecto a la excentricidad estática para elementos flexibles en el diseño por torsión sísmica estática (Tso y Wong, 1995)

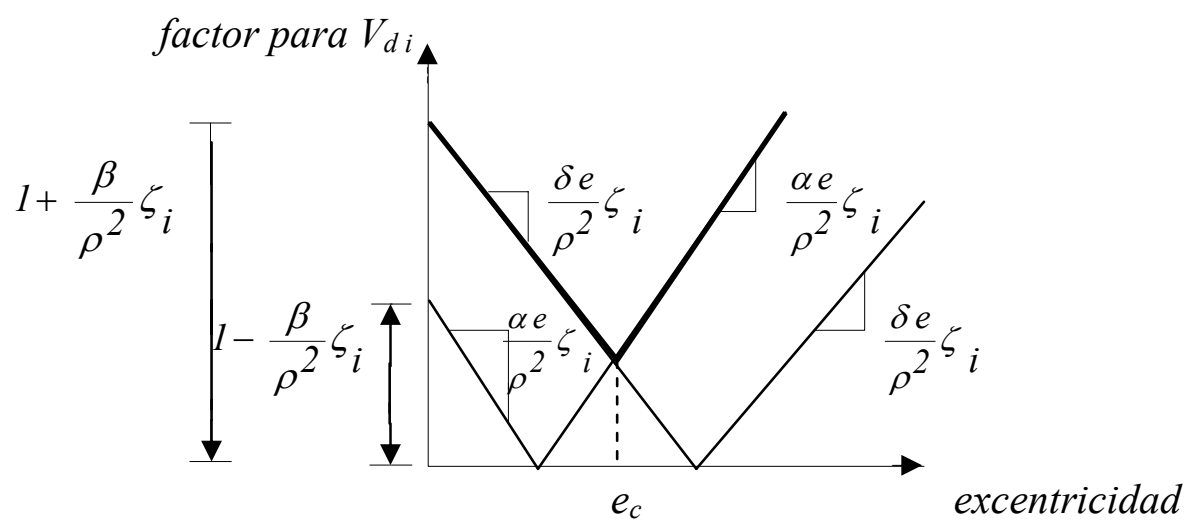

Figura 5. Representación esquemática de la variación del incremento del cortante directo con respecto a la excentricidad estática para elementos rígidos en el diseño por torsión sísmica estática (Tso y Wong, 1995) 
En las siguientes secciones se simplifica el uso de estas ecuaciones. En las figs 6 y 7 , para las ecuaciones (37) y (38) respectivamente, se presenta el comportamiento del $F A T r_{i}$ con respecto al radio de giro normalizado, para un valor de excentricidad estática normalizada igual a 0.2 , y diferentes valores de $\zeta_{i}$. En este caso se utilizaron los valores de los factores de amplificación dinámica y de excentricidad accidental que establece el RCDF.

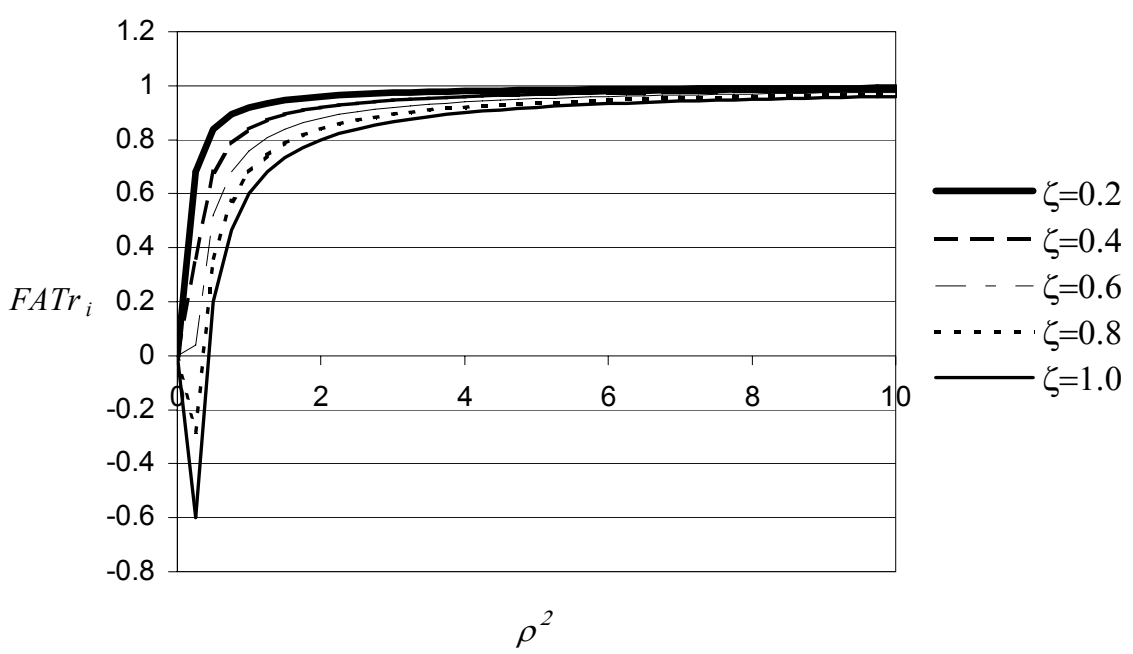

Figura 6. Variación del factor de amplificación por torsión de elementos rigidos, $F_{A T r}$ con respecto a $\rho^{2}$, para $e=0.2$, y diferentes valores de $\zeta_{i}$. Calculado con la ecuación (37).

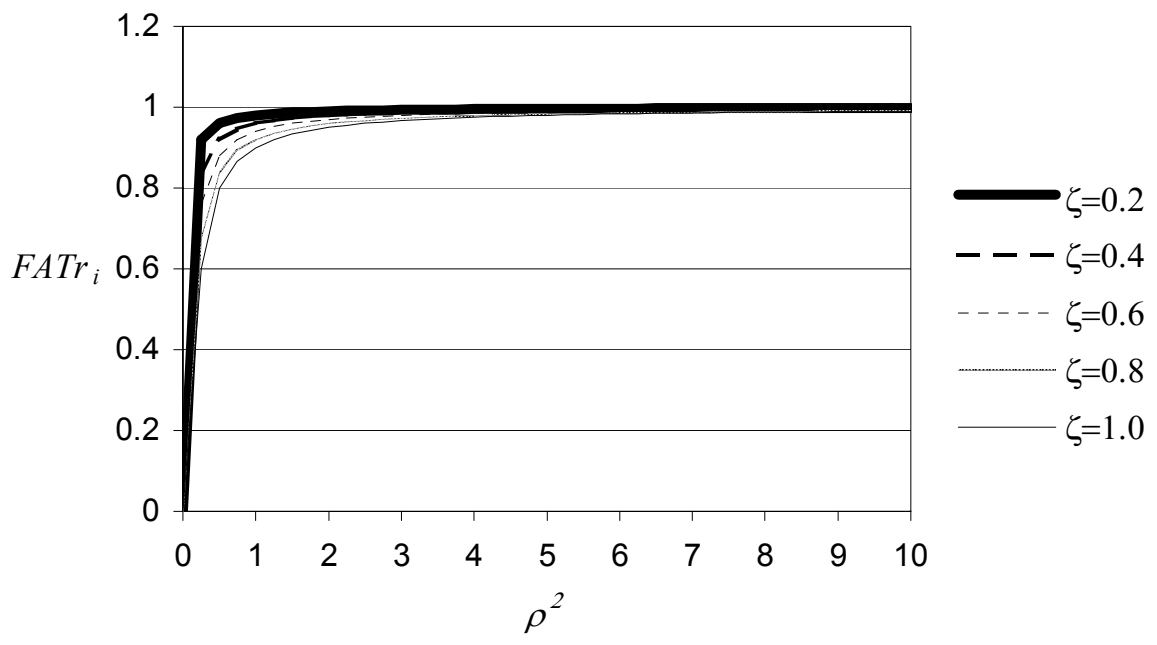

Figura 7. Variación del factor de amplificación por torsión de elementos rígidos, $F A T r_{i}$ con respecto a $\rho^{2}$, para $e=0.2$, y diferentes valores de $\zeta_{i}$. Calculado con la ecuación (38). 
En general, se puede observar que cuando el $F A T$ se evalúa con la ecuación (37), debido a los valores que intervienen en su cálculo, se puede llegar a obtener valores negativos y aun nulos. Esto es, existe el riesgo de que el valor del cortante por torsión calculado sea mayor o igual al del cortante directo. En el primer caso, los elementos resistentes probablemente se diseñarían para valores de fuerzas cortantes inferiores a las de los cortantes directos, lo cual no es realista. De acuerdo con la ecuación (37), esta condición se produciría en los elementos de los entrepisos en los que

$$
\zeta_{i}>\frac{\rho^{2}}{(\beta+\alpha e)}
$$

El caso extremo se presentaría cuando el valor del cortante de diseño que deberían resistir los elementos estructurales se anulara (fig 6), lo cual resulta en un absurdo pues aparentemente estos elementos no serían solicitados por fuerzas laterales.

Por el contrario, cuando el FAT se calcula con la ecuación (38), esta situación no se presenta, evitándose además obtener valores negativos (fig 7). Por lo que se confirma que la ecuación (23) controla el diseño de los elementos rígidos.

Es importante mencionar que, dependiendo de los valores de la excentricidad de diseño, eventualmente puede suceder que ésta presente valores que cambien de ubicación la posición nominal del $C M$ o del $C C$. Si esto ocurre, la denominación de los elementos estructurales se modificará, siendo los más afectados los elementos rígidos que, con respecto al $C T$ o al $C R$ se convertirían en elementos flexibles y se deberían diseñar como tales. Para que esto sucediera se tendría que cumplir que

$$
1 \leq\left[1+\frac{\zeta_{i}}{\rho^{2}}(\beta-\delta e)\right]
$$

de donde

$$
\delta e \leq \beta
$$

Por lo tanto, para el RCDF, los elementos rígidos, se deberán diseñar con las ecuaciones (34) y (35) siempre y cuando el valor de la excentricidad estructural normalizada, $e$, sea menor o igual que 0.1 .

Por otro lado, en la fig 7 se puede observar que, debido a los valores que pueden adoptar los parámetros que intervienen en la ecuación (38), ésta no está exenta de poder llegar a proporcionar valores en el $F A T$ menores que la unidad, lo cual produciría cortantes de diseño inferiores a los cortantes directos. Aparentemente, en la práctica del diseño por torsión sísmica estática, esta situación se trata de evitar restando un porcentaje inferior al 100\% del cortante por torsión al cortante directo en el diseño de los elementos rígidos (Damy, 1988). 


\section{Propuesta de las Normas Técnicas Complementarias para Diseño por Sismo (NTC-2001)}

En estudios recientes se ha encontrado que estructuras sometidas a cargas sísmicas intensas, pueden llegar a presentar comportamiento inadecuado al diseñarlas restando el cortante por torsión al cortante directo de los elementos rígidos (Escobar, 1994, 1997; Rojas et al., 1998; Escobar et al., 1999). Con base en estas y otras observaciones, en la nueva propuesta de las Normas Técnicas Complementarias para Diseño por Sismo (NTC-2001), se modificaron las disposiciones de diseño sísmico por torsión respecto a la edición de 1995, indicándose que: "Ningún elemento estructural tendrá una resistencia menor que la necesaria para resistir la fuerza cortante directa".

Con base en esta consideración, la ecuación (38) se aplica al diseño de los elementos rígidos, siempre y cuando el valor de la excentricidad estructural normalizada, $e$, sea menor que 0.1 , para cumplir con las consideraciones de las NTC-2001. En el caso de que $e>0.1$ :

$$
F A r_{i}=1.0
$$

En el caso de los elementos flexibles, el cortante total de diseño, calculado con la ecuación (33), resulta ser siempre mayor que el cortante directo.

\section{OBSERVACIONES SOBRE EL CORTANTE DEBIDO A LA TORSIÓN POR EXCENTRICIDAD ACCIDENTAL}

Con la ecuación (27) es posible calcular el efecto de la excentricidad accidental en los elementos estructurales. Para el $i$-ésimo elemento estructural más alejado del $C R$ ubicado en el $j$-ésimo entrepiso de una estructura, esta ecuación alcanza un valor máximo que estará dado por

$$
F E A_{\max j}=\frac{\beta}{\rho^{2} b} x_{\max i}
$$

El valor extremo que puede tener $x_{\max }$, medido desde el $C R$, será el correspondiente a la dimensión $b$ de la planta del entrepiso, en este caso la ecuación anterior se reduce a

$$
F E A_{\max j}=\frac{\beta}{\rho^{2}}
$$

Para estructuras diseñadas con el $\operatorname{RCDF}(\beta=0.1)$, en la fig 3 se presenta, para distintos valores del parámetro $\rho^{2}$, el valor máximo del Factor de Excentricidad Accidental, FEA $A_{\max } j$ calculado con la ecuación (44). Se puede apreciar que para valores de $\rho$ superiores a la unidad, el valor del $F E A_{\max j}$ es inferior a 0.1. En estos casos, el cortante por torsión debido a excentricidad accidental no excederá el $10 \%$ del cortante directo, mientras que para $\rho^{2}=0.5$, el $F E A_{\text {max }}=0.20$. En general, se puede observar que para valores de $\rho$ inferiores a la unidad, el efecto debido a la 
excentricidad accidental presenta variaciones en un intervalo muy amplio. Por lo tanto, el incremento de la resistencia debido a la excentricidad accidental no se podría representar mediante un factor de incremento único para toda la estructura. Sin embargo, si se desea simplificar aún más el manejo del efecto de la excentricidad accidental en el diseño por torsión sísmica estática, sería posible calcular el $F E A_{\max }$ para cada entrepiso y afectar con él los valores correspondientes de cortante directo de los elementos resistentes. Las implicaciones de este proceder son obvias, ya que por un lado se ahorraría tiempo al diseñar los elementos, sin embargo, al hacerlo así algunos de ellos quedarían sobre-diseñados. En una sección posterior se exploran estas opciones.

En la fig 8 se presentan diferentes casos de distribuciones en planta de elementos estructurales así como los valores del parámetro $\rho$ correspondientes.

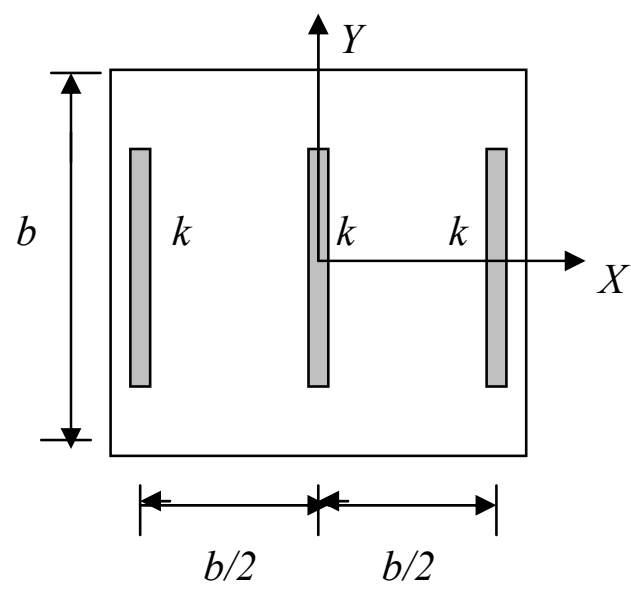

a) $\rho_{x}^{2}=0.167$

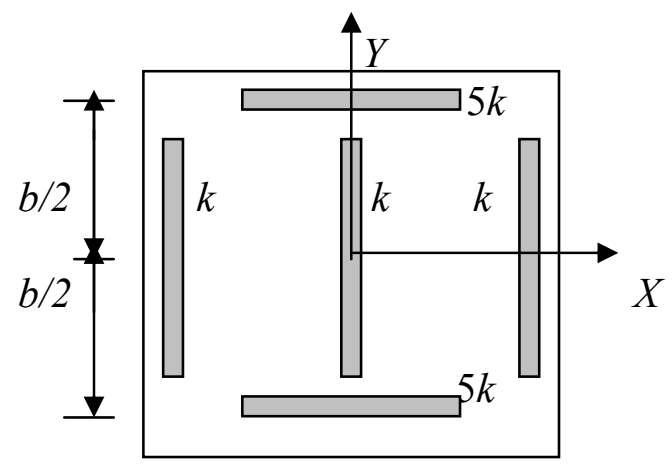

c) $\rho_{x}^{2}=1.0, \rho_{y}^{2}=0.3$

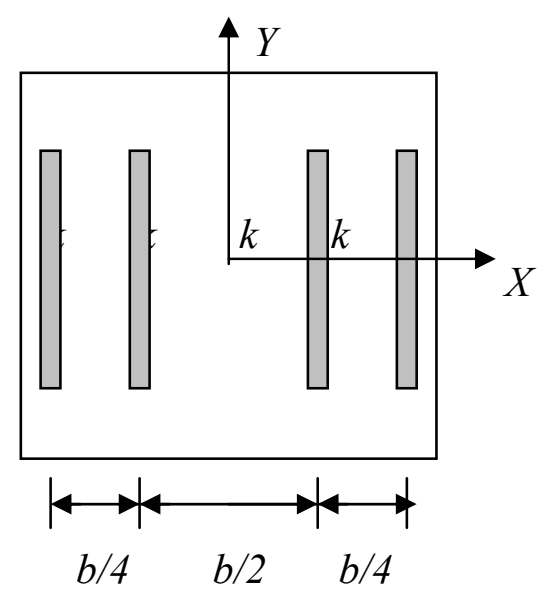

b) $\rho_{x}^{2}=0.156$

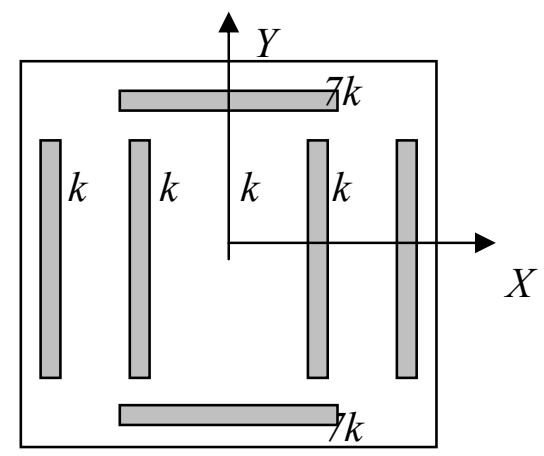

d) $\rho_{x}{ }^{2}=1.031, \rho_{y}^{2}=0.295$

Figura 8. Diferentes casos de distribución en planta de elementos resistentes y su correspondiente $\rho^{2}$. 


\section{PROCEDIMIENTO SIMPLIFICADO PARA DISTRIBUIR EL CORTANTE POR TORSIÓN SÍSMICA ESTÁTICA}

A continuación se propone el Procedimiento Simplificado de Diseño por torsión sísmica estática, $P S D$, utilizando factores de amplificación por torsión como una nueva opción para llevar a cabo el diseño por torsión sísmica. Este consta de los siguientes pasos:

1. Calcular las fuerzas cortantes de entrepiso a partir de un análisis sísmico estático considerando un sistema de fuerzas equivalentes obtenidas de un espectro de diseño sísmico.

2. Calcular los cortantes directos en los elementos estructurales. Se hace aplicando estáticamente las fuerzas calculadas en el paso anterior en los $C M$ de cada uno de los pisos de un modelo tridimensional de la estructura impidiendo los giros de las losas alrededor de un eje vertical.

3. Calcular las coordenadas de los $\boldsymbol{C} \boldsymbol{C}$ y $\boldsymbol{C R}$ de cada uno de los entrepisos con los cortantes directos utilizando las ecuaciones (10) y (11), respectivamente.

4. Calcular la excentricidad estructural, $e_{s}$, con las coordenadas del $C C$ y del $C R$ de cada uno de los entrepisos con la ecuación (9). Con estos datos, clasificar a los elementos resistentes como flexibles si se encuentran del mismo lado del $C C$ con respecto al $C R, \mathrm{y}$ como rígidos en caso contrario. Debido a esta clasificación se puede utilizar el valor absoluto de $e_{s}$ en la aplicación del método.

5. Calcular los FAT de los elementos resistentes flexibles y rígidos respectivamente, utilizando las ecuaciones (32) y (34) que para el caso del RCDF serán

$$
\begin{aligned}
& \text { FATf }_{i}=1+\frac{\zeta_{i}}{\rho^{2}}(0.1+1.5 e) \\
& \text { FATr }_{i}=1+\frac{\zeta_{i}}{\rho^{2}}(0.1-e)
\end{aligned}
$$

En estas ecuaciones, cuando se conoce la rigidez de los elementos resistentes de cada entrepiso, el cálculo del radio de giro normalizado $\rho$ se puede hacer utilizando directamente la ecuación (16). Cuando no se cuenta con estos datos el cálculo de dicho parámetro se puede determinar a partir de los cortantes directos y los desplazamientos correspondientes de entrepiso obtenidos del análisis estructural realizado en el paso 2 . Para ello, el radio de giro normalizado, $\rho_{x j}$ y $\rho_{y j}$, para cada uno de los ejes ortogonales, $X$ e $Y$, del $j$-ésimo entrepiso de la estructura se puede obtener modificando la ecuación (16) como sigue 


$$
\begin{aligned}
& \rho_{x j}=\frac{1}{b_{x j}} \sqrt{\frac{\sum V_{d y i} x_{i}^{2} / d y_{j}+\sum V_{d x i} y_{i}^{2} / d x_{j}}{\sum V_{d x i} / d x_{j}}} \\
& \rho_{y j}=\frac{1}{b_{y j}} \sqrt{\frac{\sum V_{d y i} x_{i}^{2} / d y_{j}+\sum V_{d x i} y_{i}^{2} / d x_{j}}{\sum V_{d y i} / d y_{j}}}
\end{aligned}
$$

donde $b_{x j}$ y $b_{y j}$ son las dimensiones máximas en planta del entrepiso, perpendiculares a la dirección de excitación sísmica; y $V_{d x}, V_{d y} i$ y $d x_{j}, d y_{j}$ son los cortantes directos del $i$ ésimo elemento resistente y los desplazamiento relativos de entrepiso correspondientes obtenidos del análisis estructural estático realizado para obtener los cortantes directos en los elementos estructurales.

6. Calcular las fuerzas de diseño en los elementos estructurales. Para obtener las fuerzas de diseño, las fuerzas en los elementos estructurales (momentos flexionantes, fuerzas axiales y cortantes, etc.) producidas por los cortantes directos calculados en el paso 2, se multiplican por los correspondientes $F A T$ calculados en el paso anterior.

\section{APLICACIÓN}

Para ilustrar y comparar la aplicación del procedimiento propuesto, se utiliza el edificio de cinco pisos estudiado por Bazán y Meli (1998), mostrado en la fig 1.

\section{Fuerzas cortantes de entrepiso}

Se calculan a partir de las fuerzas laterales provenientes de un análisis sísmico estático considerando un sistema de fuerzas equivalentes obtenidas de un espectro de diseño sísmico. De acuerdo con Bazán y Meli (1998), las fuerzas laterales producidas en dirección $X$ se presentan en la tabla 1.

\section{Cortantes directos en los elementos estructurales}

Al conocer la rigidez de cada uno de los elementos resistentes, el cortante directo sobre cada uno de ellos se puede determinar aplicando la ecuación (1). También, con un modelo tridimensional de la estructura, mediante un programa de análisis estructural, las fuerzas laterales de la tabla 1 se aplicaron actuando estáticamente en dirección $X$ en el $C M$ de cada uno de los pisos de la estructura impidiendo los giros de las losas alrededor de un eje vertical, y permitiendo únicamente el desplazamiento lateral correspondiente. Las fuerzas cortantes resultantes obtenidas en cada uno de los elementos resistentes son proporcionales a su rigidez. En las tablas 3 y 4 , se presentan los cortantes directos generados en cada uno de los elementos resistentes del entrepiso cinco para las direcciones de análisis $X$ e $Y$, respectivamente. 
Tabla 3. Cálculo de la excentricidad estructural. Dirección $X$.

\begin{tabular}{|c|c|c|c|c|c|c|c|c|c|}
\hline $\begin{array}{c}\text { Entre- } \\
\text { piso }\end{array}$ & $V_{x}$ & \multicolumn{4}{|c|}{ Cortante directo $(\mathrm{t})$} & $y_{C C}$ & $y_{C R}$ & $e_{s}=\left|y_{C C}-y_{C R}\right|$ & $e$ \\
\cline { 3 - 6 } & $(\mathrm{t})$ & $V_{d l x}$ & $V_{d 2 x}$ & $V_{d 3 x}$ & $V_{d 4 x}$ & $(\mathrm{~m})$ & $(\mathrm{m})$ & $(\mathrm{m})$ & \\
\hline 5 & 23.77 & 6.48 & 4.32 & 12.97 & --- & 3.75 & 4.73 & 0.98 & 0.13 \\
\hline 4 & 49.52 & 13.51 & 9.00 & 9.00 & 18.01 & 4.66 & 6.00 & 1.34 & 0.122 \\
\hline 3 & 74.28 & 21.85 & 13.11 & 13.11 & 26.22 & 4.94 & 5.82 & 0.88 & 0.080 \\
\hline 2 & 91.61 & 26.95 & 16.17 & 16.17 & 32.33 & 5.05 & 5.82 & 0.77 & 0.071 \\
\hline 1 & 103.50 & 30.44 & 18.26 & 18.26 & 36.53 & 5.19 & 5.82 & 0.63 & 0.058 \\
\hline
\end{tabular}

Tabla 4. Cálculo de la excentricidad estructural. Dirección $Y$.

\begin{tabular}{|c|c|c|c|c|c|c|c|c|c|}
\hline \multirow{2}{*}{$\begin{array}{l}\text { Entre- } \\
\text { piso }\end{array}$} & \multirow{2}{*}{$\begin{array}{l}V_{y} \\
(\mathrm{t})\end{array}$} & \multicolumn{4}{|c|}{ Cortante directo $(\mathrm{t})$} & \multirow{2}{*}{$\begin{array}{l}x_{C C} \\
\text { (m) }\end{array}$} & \multirow{2}{*}{$\begin{array}{l}x_{C R} \\
(\mathrm{~m})\end{array}$} & \multirow{2}{*}{$\begin{array}{c}e_{s}=\left|x_{C C^{-}} x_{C R}\right| \\
(\mathrm{m})\end{array}$} & \multirow[t]{2}{*}{$e$} \\
\hline & & $V_{d l v}$ & $V_{d 2 v}$ & $V_{d 3 v}$ & $V_{d 4 v}$ & & & & \\
\hline 5 & 47.54 & 26.45 & 1.43 & 19.66 & --- & 6.75 & 5.78 & 0.97 & 0.072 \\
\hline 4 & 99.04 & 51.92 & 2.88 & 2.88 & 41.35 & 8.02 & 8.93 & 0.91 & 0.045 \\
\hline 3 & 148.56 & 80.58 & 3.78 & 3.78 & 60.43 & 8.42 & 8.64 & 0.23 & 0.011 \\
\hline 2 & 183.23 & 99.38 & 4.66 & 4.66 & 74.53 & 8.56 & 8.64 & 0.08 & 0.004 \\
\hline 1 & 207.0 & 112.27 & 5.26 & 5.26 & 84.20 & 8.56 & 8.64 & 0.09 & 0.004 \\
\hline
\end{tabular}

\section{Posición del $C R$ de los entrepisos}

Con los cortantes directos se calculan las coordenadas del $C R$ de cada entrepiso, en el Apéndice B se anotan los cálculos para el entrepiso cinco. En las tablas 3 y 4 se presenta la localización de los $C R$ y de los $C C$ para las direcciones de análisis $X$ e $Y$ de la estructura, respectivamente.

\section{Excentricidad estructural}

Utilizando las coordenadas de los $C R$ y los $C C$ de cada entrepiso se calcula la excentricidad estructural correspondiente. En las tablas 3 y 4 se muestran los valores calculados.

\section{Cálculo de los factores de amplificación por torsión}

Para su diseño, cada uno de los elementos estructurales se clasifica de acuerdo con su posición respecto al $C R$ y al $C C$ de cada entrepiso. Los FAT se calculan con las ecuaciones (32) y (34) como se especifica en el paso 5 del procedimiento. En las tablas 5 y 6 se presenta la clasificación y ubicación de los elementos estructurales del entrepiso cinco del edificio estudiado, correspondientes a las direcciones $X$ e $Y$ respectivamente y en el Apéndice B se muestran los cálculos.

\section{Cálculo de los efectos de torsión sobre los elementos resistentes}

En las tablas 5 y 6 se presentan los FAT para cada uno de los elementos resistentes del entrepiso cinco para las direcciones $X$ e $Y$ de análisis. También se presentan los cortantes totales, incluyendo la torsión, calculados con el $P S D$ propuesto. En la última columna de estas tablas se anotan los cortantes totales obtenidos por Bazán y Meli, se puede observar que estos valores son prácticamente iguales a los obtenidos con el procedimiento aquí propuesto. 
Tabla 5. Cortantes totales en los elementos resistentes. Dirección $X$, entrepiso cinco.

\begin{tabular}{|c|c|c|c|c|c|c|}
\hline Marco & Tipo & $\zeta_{i}$ & $V_{d}$ & $F A T_{i}$ & \multicolumn{2}{|c|}{ Cortante total (t) } \\
\cline { 6 - 7 } & & & $(\mathrm{t})$ & & $P S D$ & Bazán-Meli \\
\hline $1 \mathrm{X}$ & flexible & 0.630 & 6.48 & 1.074 & 6.96 & 6.97 \\
\hline $2 \mathrm{X}$ & flexible & 0.164 & 4.32 & 1.019 & 4.41 & 4.41 \\
\hline $3 \mathrm{X}$ & rígido & 0.370 & 12.97 & 0.996 & 12.91 & 12.90 \\
\hline
\end{tabular}

Tabla 6. Cortantes totales en los elementos resistentes. Dirección $Y$, entrepiso cinco.

\begin{tabular}{|c|c|c|c|c|c|c|}
\hline Marco & Tipo & $\zeta_{i}$ & $V_{d}$ & $F A T_{i}$ & \multicolumn{2}{|c|}{ Cortante total (t) } \\
\cline { 6 - 7 } & & & $(\mathrm{t})$ & & $P S D$ & Bazán-Meli \\
\hline 1Y & rígido & 0.428 & 26.45 & 1.047 & 27.69 & 27.09 \\
\hline 2Y & flexible & 0.053 & 1.43 & 1.043 & 1.49 & 1.46 \\
\hline $3 \mathrm{Y}$ & flexible & 0.572 & 19.66 & 1.464 & 28.78 & 28.19 \\
\hline
\end{tabular}

En las tablas 7 y 8 se presentan los valores de las fuerzas de diseño en columnas del entrepiso cinco, obtenidas con el $P S D$ y con el método tradicional y sus errores relativos, respectivamente. Como se puede observar, los valores de las fuerzas son prácticamente los mismos, con errores relativos inferiores al $1 \%$, y pueden atribuirse a errores de redondeo en las operaciones aritméticas.

Tabla 7. Fuerzas de diseño ( $t$ y $t-m)$, en columnas del entrepiso 5 del edificio de cinco pisos, obtenidas con el $P S D$ y con el método tradicional.

\begin{tabular}{|c|c|c|c|c|c|c|c|c|c|c|}
\hline \multirow{2}{*}{$\begin{array}{c}\text { Col. } \\
\text { en } \\
\text { ejes }\end{array}$} & \multicolumn{3}{|c|}{$\begin{array}{l}\text { Efectos del cortante } \\
\text { directo }\end{array}$} & \multirow[t]{2}{*}{$F A T$} & \multicolumn{3}{|c|}{$P S D$} & \multicolumn{3}{|c|}{ Método tradicional } \\
\hline & $V$ & Minf & Msup & & $V$ & Minf & $M \sup$ & $V$ & Minf & M sup \\
\hline $1 \mathrm{X}, 1 \mathrm{Y}$ & 2.15 & 3.21 & 3.25 & \multirow{3}{*}{1.074} & 2.31 & 3.45 & 3.49 & 2.28 & 3.38 & 3.45 \\
\hline $1 \mathrm{X}, 2 \mathrm{Y}$ & 2.23 & 3.34 & 3.36 & & 2.40 & 3.59 & 3.61 & 2.38 & 3.56 & 3.59 \\
\hline $1 \mathrm{X}, 3 \mathrm{Y}$ & 2.17 & 3.26 & 3.25 & & 2.33 & 3.50 & 3.49 & 2.31 & 3.47 & 3.46 \\
\hline $2 \mathrm{X}, 1 \mathrm{Y}$ & 1.45 & 2.16 & 2.18 & \multirow{3}{*}{1.019} & 1.48 & 2.20 & 2.22 & 1.45 & 2.16 & 2.18 \\
\hline $2 \mathrm{X}, 2 \mathrm{Y}$ & 1.49 & 2.23 & 2.24 & & 1.52 & 2.27 & 2.28 & 1.49 & 2.22 & 2.23 \\
\hline $2 \mathrm{X}, 3 \mathrm{Y}$ & 1.48 & 2.22 & 2.20 & & 1.51 & 2.26 & 2.24 & 1.48 & 2.22 & 2.22 \\
\hline $3 \mathrm{X}, 1 \mathrm{Y}$ & 4.15 & 6.20 & 6.23 & \multirow{3}{*}{0.996} & 4.13 & 6.18 & 6.21 & 4.15 & 6.19 & 6.27 \\
\hline $3 \mathrm{X}, 2 \mathrm{Y}$ & 4.40 & 6.58 & 6.61 & & 4.38 & 6.55 & 6.58 & 4.48 & 6.70 & 6.75 \\
\hline $3 X, 3 Y$ & 4.25 & 6.44 & 6.33 & & 4.23 & 6.41 & 6.30 & 4.25 & 6.41 & 6.35 \\
\hline
\end{tabular}

Tabla 8. Errores relativos (\%) con respecto al método tradicional de diseño, de las fuerzas en columnas del edificio de cinco pisos, obtenidas con el $P S D$.

\begin{tabular}{|c|c|c|c|c|c|c|c|}
\hline \multirow{2}{*}{$\begin{array}{c}\text { Entre- } \\
\text { piso }\end{array}$} & Col. en & \multicolumn{3}{|c|}{ Sismo en dirección $X$} & \multicolumn{3}{c|}{ Sismo en dirección $Y$} \\
\cline { 3 - 8 } & ejes & $V$ & $M$ inf & $M$ sup & $V$ & $M$ inf & $M$ sup \\
\hline 5 & $2 \mathrm{X}, 3 \mathrm{Y}$ & 0.0 & 0.0 & 0.0 & 0.0 & 0.0 & 0.2 \\
\hline 3 & $2 \mathrm{X}, 3 \mathrm{Y}$ & 0.0 & 0.0 & 0.1 & 0.0 & 0.9 & 0.9 \\
\hline 1 & $1 \mathrm{X}, 1 \mathrm{Y}$ & 0.0 & 0.0 & 0.0 & 0.0 & 0.0 & 0.0 \\
\hline
\end{tabular}




\section{Criterio de diseño de la propuesta de las Normas Técnicas Complementarias para Diseño por Sismo (NTC-01)}

La aplicación del procedimiento de diseño simplificado al utilizar la propuesta de las Normas Técnicas Complementarias para Diseño por Sismo (NTC-2001), modifica el diseño de los elementos rígidos. Así, para la dirección $X$ del entrepiso cinco del edificio analizado se tiene que:

$e=0.13>0.1$

Por lo que, de la ecuación (42), el FAT para el marco 3X será igual a la unidad, y el cortante sísmico total será el igual cortante directo, esto es:

$V_{3 x}=V_{d 3 x}=12.97 t$

En la dirección $Y$ de análisis, para el mismo entrepiso, los cálculos para el cortante total no se modifican ya que $e=0.071<0.1$. En las tablas 9 y 10 se muestran los cortantes totales calculados con el $P S D$ propuesto y los obtenidos por Bazán y Meli (1998) para cada una de las direcciones analizadas y para todos los elementos resistentes de los entrepisos del edificio. En estas tablas se presentan también los cortantes por torsión corregidos con las disposiciones de la propuesta NTC-2001. En particular, en los elementos rígidos 4X y 3X de los entrepisos 4 y 5 respectivamente, la aplicación de estas disposiciones, aunque en este caso en un porcentaje inferior a $1 \%$, evita reducir el valor del cortante de diseño.

Tabla 9. Cortantes totales en los elementos resistentes. Dirección $X$.

\begin{tabular}{|c|c|c|c|c|}
\hline Entre- & Marco, & \multicolumn{3}{|c|}{ Cortantes totales (t) } \\
\cline { 2 - 5 } Piso & tipo & $P S D$ & Bazán-Meli & NTC-2001 \\
\hline \multirow{4}{*}{5} & $1 \mathrm{X}, \mathrm{f}$ & 6.96 & 6.97 & 6.96 \\
\cline { 2 - 5 } & $2 \mathrm{X}, \mathrm{f}$ & 4.40 & 4.41 & 4.40 \\
\cline { 2 - 5 } & $3 \mathrm{X}, \mathrm{r}$ & 12.91 & 12.90 & 12.97 \\
\hline \multirow{4}{*}{4} & $1 \mathrm{X}, \mathrm{f}$ & 14.05 & 14.07 & 14.05 \\
\cline { 2 - 5 } & $2 \mathrm{X}, \mathrm{f}$ & 9.16 & 9.17 & 9.16 \\
\cline { 2 - 5 } & $3 \mathrm{X}, \mathrm{r}$ & 9.00 & 9.01 & 9.00 \\
\cline { 2 - 5 } & $4 \mathrm{X}, \mathrm{r}$ & 17.96 & 17.98 & 18.01 \\
\hline \multirow{4}{*}{3} & $1 \mathrm{X}, \mathrm{f}$ & 22.74 & 22.73 & 22.74 \\
\cline { 2 - 5 } & $2 \mathrm{X}, \mathrm{f}$ & 13.32 & 13.29 & 13.32 \\
\cline { 2 - 5 } & $3 \mathrm{X}, \mathrm{r}$ & 13.12 & 13.09 & 13.12 \\
\cline { 2 - 5 } & $4 \mathrm{X}, \mathrm{r}$ & 26.30 & 26.31 & 26.30 \\
\hline \multirow{4}{*}{2} & $1 \mathrm{X}, \mathrm{f}$ & 27.97 & & 27.97 \\
\cline { 2 - 5 } & $2 \mathrm{X}, \mathrm{f}$ & 16.41 & & 16.41 \\
\cline { 2 - 5 } & $3 \mathrm{X}, \mathrm{r}$ & 16.19 & & 16.19 \\
\cline { 2 - 5 } & $4 \mathrm{X}, \mathrm{r}$ & 32.49 & & 32.49 \\
\hline \multirow{3}{*}{1} & $1 \mathrm{X}, \mathrm{f}$ & 31.49 & & 31.49 \\
\cline { 2 - 5 } & $2 \mathrm{X}, \mathrm{f}$ & 18.52 & & 18.52 \\
\cline { 2 - 5 } & $3 \mathrm{X}, \mathrm{r}$ & 18.31 & & 36.31 \\
\cline { 2 - 5 } & $4 \mathrm{X}, \mathrm{r}$ & 36.78 & & \\
\hline
\end{tabular}

( $f=$ elemento flexible, $r=$ elemento rígido) 


\section{OBSERVACIONES SOBRE LA TORSIÓN DEBIDA A EXCENTRICIDAD ACCIDENTAL}

En la columna 2 de la tabla 11 se anotan estos valores de los $F E A$ de los elementos estructurales para el entrepiso cinco del edificio estudiado calculados con la ecuación (27). Además, debido a que el efecto de la excentricidad accidental sobre los elementos estructurales siempre es aditivo, si se deseara simplificar aún más el manejo del efecto de la excentricidad accidental en el diseño por torsión sísmica estática, una forma de hacerlo consistiría en calcular el $F E A_{\max } j$ para cada entrepiso y afectar con él los valores correspondientes de cortante directo de los elementos resistentes del mismo. Esto es, para la dirección $X$

$F E A_{\max 5}=\frac{0.1}{2.5119}=0.0398$

Para la dirección $Y$

$F E A_{\max 5}=\frac{0.1}{0.2565}=0.389$

Tabla 10. Cortantes totales en los elementos resistentes. Dirección $Y$.

\begin{tabular}{|c|c|c|c|c|}
\hline \multirow{2}{*}{$\begin{array}{c}\text { Entre- } \\
\text { Piso }\end{array}$} & \multirow{2}{*}{$\begin{array}{c}\text { Marco, } \\
\text { tipo }\end{array}$} & \multicolumn{3}{|c|}{ Cortantes totales $(\mathrm{t})$} \\
\hline & & $P S D$ & Bazán-Meli & NTC-2001 \\
\hline \multirow{3}{*}{5} & $1 \mathrm{Y}, \mathrm{r}$ & 27.69 & 27.09 & 27.69 \\
\hline & $2 Y, f$ & 1.49 & 1.46 & 1.49 \\
\hline & $3 \mathrm{Y}, \mathrm{f}$ & 28.78 & 28.19 & 28.78 \\
\hline \multirow{4}{*}{4} & $1 \mathrm{Y}, \mathrm{f}$ & 67.82 & 67.80 & 67.82 \\
\hline & $2 \mathrm{Y}, \mathrm{f}$ & 3.12 & 3.11 & 3.12 \\
\hline & $3 \mathrm{Y}, \mathrm{r}$ & 3.02 & 3.02 & 3.02 \\
\hline & $4 \mathrm{Y}, \mathrm{r}$ & 46.40 & 46.38 & 46.40 \\
\hline \multirow{4}{*}{3} & $1 \mathrm{Y}, \mathrm{f}$ & 96.85 & 96.80 & 96.85 \\
\hline & $2 Y, f$ & 3.97 & 3.90 & 3.97 \\
\hline & $3 \mathrm{Y}, \mathrm{r}$ & 4.10 & 4.04 & 4.10 \\
\hline & $4 \mathrm{Y}, \mathrm{r}$ & 72.70 & 72.72 & 72.70 \\
\hline \multirow{4}{*}{2} & $1 \mathrm{Y}, \mathrm{f}$ & 117.64 & & 117.64 \\
\hline & $2 \mathrm{Y}, \mathrm{f}$ & 4.87 & & 4.87 \\
\hline & $3 \mathrm{Y}, \mathrm{r}$ & 5.09 & & 5.09 \\
\hline & $4 \mathrm{Y}, \mathrm{r}$ & 90.83 & & 90.83 \\
\hline \multirow{4}{*}{1} & $1 \mathrm{Y}, \mathrm{f}$ & 133.01 & & 133.01 \\
\hline & $2 \mathrm{Y}, \mathrm{f}$ & 5.50 & & 5.50 \\
\hline & $3 \mathrm{Y}, \mathrm{r}$ & 5.75 & & 5.75 \\
\hline & $4 Y, r$ & 102.55 & & 102.55 \\
\hline
\end{tabular}

$(\mathrm{f}=$ elemento flexible, $\mathrm{r}=$ elemento rígido $)$

En la columna 3 de la tabla 11 se anotan los valores de los FAT calculados aplicando la ecuación (30) con los valores de los $F E A_{\max } 5$, para la dirección $X$ y $Y$. En la columna 4 de esta tabla se anota el incremento de resistencia que se obtendría al diseñar los elementos estructurales 
con estos FAT. Se observa que los mayores valores se presentan en los elementos ubicados en dirección $Y$.

Tabla 11. Factores de excentricidad accidental y de amplificación por torsión, entrepiso cinco, dirección $X$.

\begin{tabular}{|c|c|c|c|c|c|}
\hline Marco & $\begin{array}{c}F E A_{i} \\
\text { Ec.(17) }\end{array}$ & $\begin{array}{c}F A T_{i} \text { con } \\
F E A_{\max } \text { 5 }\end{array}$ & $\begin{array}{c}\text { Sobre- } \\
\text { resist. }\end{array}$ & $\begin{array}{c}F A T_{i} \text { con } \\
F E A_{I}=1.1\end{array}$ & $\begin{array}{c}\text { Sobre- } \\
\text { resist. }\end{array}$ \\
\hline $1 \mathrm{X}$ & 0.025 & 1.089 & 1.01 & 1.15 & 1.07 \\
\hline $2 \mathrm{X}$ & 0.007 & 1.054 & 1.03 & 1.11 & 1.09 \\
\hline $3 \mathrm{X}$ & 0.015 & 1.021 & 1.03 & 1.08 & 1.09 \\
\hline $1 \mathrm{Y}$ & 0.167 & 1.269 & 1.21 & -- & -- \\
\hline $2 \mathrm{Y}$ & 0.021 & 1.411 & 1.35 & -- & -- \\
\hline $3 \mathrm{Y}$ & 0.223 & 1.629 & 1.11 & -- & -- \\
\hline
\end{tabular}

Otra idea para simplificar el manejo de la torsión debido a la excentricidad accidental en el diseño por torsión sísmica estática sería utilizando los resultados mostrados en la fig 3. En ella se puede apreciar que para valores de $\rho$ iguales o mayores que la unidad, el valor del incremento del cortante directo debido a excentricidad accidental no excederá el 10\% del cortante directo. En estos casos $F E A=0.1$ para todos los elementos resistentes del entrepiso orientados en la dirección correspondiente. Para el caso contrario en el que $\rho<1$, entonces se seguiría el PSD propuesto. En las columnas 5 y 6 de la tabla 11 se anotan los valores de los FAT calculados con este criterio, así como los valores en el incremento de la resistencia que se produciría en los elementos estructurales al diseñarlos de esta forma.

En estas dos últimas opciones, para simplificar el manejo de la torsión debido a la excentricidad accidental en el diseño por torsión sísmica estática, el efecto de ésta se incluye a través de un factor de incremento único para todos los elementos resistentes de cada entrepiso de la estructura. Sin embargo, debido a que este incremento no es constante, el costo de esta simplificación ocasionaría que algunos de ellos estuvieran sobre diseñados hasta en $35 \%$ en la primera opción y en $9 \%$ en la segunda.

\section{CONCLUSIONES Y RECOMENDACIONES}

En el presente trabajo se propone un método denominado Procedimiento Simplificado de Diseño, $P S D$, que simplifica el diseño por torsión sísmica estática de edificios. Para ello se estudió la distribución de las fuerzas cortantes entre los elementos resistentes de entrepiso en edificios diseñados por torsión sísmica. Se analizaron los factores que afectan la excentricidad de diseño, particularmente la excentricidad accidental, con el objetivo de simplificar su manejo en el criterio de diseño por torsión sísmica del RCDF vigente. La principal motivación a la que obedece esta propuesta es que, al simplificar el procedimiento de diseño por torsión, se reduzca el número de análisis al incluir la excentricidad estructural en modelos tridimensionales.

Con el análisis estructural que se hace del edificio para calcular los centros de torsión, se obtienen las fuerzas causadas por los cortantes directos en los elementos estructurales (momentos flexionantes, fuerzas cortantes, axiales, etc.). Con el $P S D$ propuesto, el método de diseño por 
torsión sísmica estática se reduce a multiplicar el valor de estas fuerzas por su correspondiente Factor de Amplificación por Torsión, FAT, para obtener las fuerzas de diseño. Por otro lado, con la clasificación de elementos rígidos y flexibles necesaria para aplicar el PSD, es posible tener una mejor interpretación de la distribución de las fuerzas entre los elementos resistentes al utilizar una ecuación correspondiente a cada tipo de ellos.

Se demostró que el efecto de la torsión, debido a la excentricidad accidental, siempre es aditivo en los elementos estructurales, y que depende de su posición con respecto al centro de torsión y del radio de giro normalizado.

Con el procedimiento propuesto se obtuvieron prácticamente los mismos resultados que con el método tradicional, las diferencias observadas se pueden atribuir a las operaciones aritméticas que interviene en los cálculos.

Se exploraron otras dos posibilidades para incluir el efecto de la excentricidad accidental en el diseño por torsión sísmica estática a través de un factor de incremento único. En la segunda de ellas se encontró que el valor de este factor es igual al 10\% del cortante directo para cada uno de los elementos de los entrepisos donde se cumpla que el radio de giro normalizado sea mayor o igual que la unidad. Sin embargo, debido a que, como se demostró, el valor real de este factor no es constante, el costo de esta sobre simplificación, para los casos estudiados, ocasionaría que algunos de los elementos estructurales estuvieran sobre-diseñados hasta en $9 \%$. Sin embargo, debido a que el RCDF no especifica un límite superior para la resistencia de los elementos estructurales, esta alternativa se podría manejar como opcional, aunque se recomienda realizar estudios más detallados (Giorgana, 2003).

Finalmente, con el PSD propuesto, es suficiente un solo análisis en cada una de las direcciones ortogonales del modelo tridimensional de la estructura, para calcular las fuerzas de diseño incluyendo los efectos de torsión en todos los elementos estructurales.

\section{AGRADECIMIENTOS}

A Juan Dyer, Santiago Loera y David Murià sus valiosos comentarios y la revisión crítica del presente escrito. Los comentarios de los revisores contribuyeron a aclarar el contenido del artículo. La etapa inicial de este estudio fue patrocinada por la Dirección General de Obras del Gobierno del Distrito Federal.

\section{REFERENCIAS}

Bazán E, y R Meli (1998), Diseño sísmico de edificios, editorial Limusa, México.

Cheung, V W-T y W K Tso (1986), "Eccentricity in irregular multistorey buildings", Canadian Journal of Civil Engineering, Vol. 13, No. 1, pp. 46-52.

Damy, J (1988), "Comentarios al inciso 8.6 de las Normas Técnicas Complementarias para Diseño por Sismo”, Revista de Ingeniería Sísmica, SMIS, No. 33, pp. 66-99. 
De la Llera, J C y A K Chopra (1994a), "Accidental-torsion in buildings due to stiffness uncertainty", Earthquake Engineering and Structural Dynamics, Vol. 23, pp.117-136.

De la Llera, J C y A K Chopra (1994b), "Evaluation of code accidental-torsion provisions from building records", ASCE Journal of Structural Engineering, Vol. 120, pp.597-616.

Elorduy, J y E Rosenblueth (1968), "Torsiones sísmicas en edificios de un piso", Memorias, II Congreso Nacional de Ingeniería Sísmica, Veracruz, Ver., México.

Escobar, J A (1994), "Respuesta sísmica de estructuras asimétricas inelásticas con propiedades inciertas", Tesis doctoral, División de Estudios de Posgrado de la Facultad de Ingeniería, UNAM, México.

Escobar, J A (1996), "Seismic torsion in non-linear nominally symmetric structures due to random properties", Memorias, 11th World Conference on Earthquake Engineering, Acapulco Gro., México, CDROM.

Escobar, J A (1997), "Relación entre rigidez y resistencia debido a criterios de diseño por torsión”, Séptimas Jornadas Chilenas de Sismología e Ingeniería Antisísmica y Primer Congreso Iberoamericano de Ingeniería Sísmica, La Serena, Chile, Vol. I, pp. 457-464.

Escobar, J A y G Ayala (1998), "Yielding seismic response of code designed single storey asymmetric structures", Earthquake Engineering and Structural Dynamics, Vol. 27, pp. 525-541.

Escobar, J A, R Gómez y A Páez (1999), “Evaluación de criterios de diseño por torsión”, Informe para la Dirección General de Obras del Gobierno del Distrito Federal, Instituto de Ingeniería, UNAM.

Giorgana, S (2003), "La excentricidad accidental en el diseño por torsión sísmica estática”, Tesis de Ingeniero Civil en desarrollo, Facultad de Ingeniería, UNAM.

NTC-1987 (1987), "Normas Técnicas Complementarias para Diseño por Sismo", Gaceta Oficial del Departamento del Distrito Federal, 5 de noviembre de 1987.

NTC-1995 (1995), "Normas Técnicas Complementarias para Diseño por Sismo”, Gaceta Oficial del Departamento del Distrito Federal, 7 de noviembre de 1994.

NTC-2001 (2001), "Propuesta de Normas Técnicas Complementarias para Diseño por Sismo", http://www.smie.org.mx/articulos, abril.

Padilla, A (2002), "Evaluación de un requisito complementario a los criterios de diseño por torsión del RCDF en sus ediciones 1995 y 2001”, Tesis de maestría, DEPFI, UNAM.

Paulay, T (1997), “Are existing seismic provisions achieving the design aims?", Earthquake Spectra, Vol. 13, No. 2, pp. 259-279.

Pekau, O A y R Guimond (1988), "Accidental torsion in yielding symmetric structures", Memorias, 9th World Conference on Earthquake Engineering, Tokio, Japón, Vol. V, pp. V-85 a V90.

Rojas, D, J A Escobar y R Gómez (1998), "El método de componentes principales aplicado al estudio de torsión sísmica de edificios", Memorias, XI Congreso Nacional de Ingeniería Estructural, Monterrey, México, Vol. 1, pp. 521-530. 
Rosenblueth, E (1979), "Seismic design requirements in a 1976 Mexican Code", Earthquake Engineering and Structural Dynamics, Vol. 7, pp. 49-61.

Tso, W K (1990), "Static eccentricity concept for torsional moment estimations", ASCE Journal of Structural Engineering, Vol. 16, pp. 1199-1212.

Tso, W K y C M Wong (1993), "An evaluation of the New Zealand Code torsional provision", Bulletin of the New Zealand National Society for Earthquake Engineering, Vol. 26, No. 2, pp. 194207.

Tso, W K y C M Wong (1995), “Eurocode 8 seismic torsional provisions evaluation”, Journal of European Earthquake Engineering, Vol. 1, pp. 23-33.

\section{APÉNDICE A. CÁLCULO DE LA EXCENTRICIDAD DE PISO Y DE ENTREPISO PARA LA DIRECCIÓN $X$ DEL EDIFICIO DE CINCO PISOS}

Utilizando los dos conceptos de excentricidad para edificios de varios pisos, se presentan los cálculos de los $C T$ y $C R$ de piso y de entrepiso así como los momentos torsionantes obtenidos para la dirección $X$ del edifico estudiado por Bazán y Meli (1998).

Tabla A1. Cortantes y fuerzas en los elementos resistentes.

\begin{tabular}{|c|c|c|c|c|c|c|c|c|}
\hline Entrepiso & \multicolumn{3}{|c|}{ Cortante directo (t) } & \multicolumn{4}{c|}{ Fuerza (t) } \\
\hline Piso & $V_{d l x}$ & $V_{d 2 x}$ & $V_{d 3 x}$ & $V_{d 4 x}$ & $F_{l x}$ & $F_{2 x}$ & $F_{3 x}$ & $F_{4 x}$ \\
\hline 5 & 6.48 & 4.32 & 12.97 & --- & & & & \\
\hline 5 & & & & & 6.48 & 4.32 & 12.97 & --- \\
\hline 4 & 13.51 & 9.00 & 9.00 & 18.01 & & & & \\
\hline 4 & & & & & 7.03 & 4.68 & -3.97 & 18.01 \\
\hline 3 & 21.85 & 13.11 & 13.11 & 26.22 & & & & \\
\hline 3 & & & & & 8.34 & 4.11 & 4.11 & 8.21 \\
\hline 2 & 26.94 & 16.17 & 16.17 & 32.33 & & & & \\
\hline 2 & & & & & 5.09 & 3.06 & 3.06 & 6.11 \\
\hline 1 & 30.11 & 18.26 & 18.26 & 36.53 & & & & \\
\hline 1 & & & & & 3.50 & 2.09 & 2.09 & 4.20 \\
\hline
\end{tabular}


Cálculos para la excentricidad de piso. Coordenadas de los $C T$, ecuación (6)

$$
\begin{aligned}
& y_{C T 5}=\frac{6.48(0)+4.32(3.5)+12.97(7.5)}{23.77}=4.73 \mathrm{~m} \\
& y_{C T 4}=\frac{7.03(0)+4.68(3.5)+(-3.97)(7.5)+18.01(11)}{25.75}=7.17 \mathrm{~m} \\
& y_{C T 3}=\frac{8.34)(0)+4.11(3.5)+4.11(7.5)+8.21(11)}{24.76}=5.47 \mathrm{~m} \\
& y_{C T 2}=\frac{5.09(0)+3.06(3.5)+3.06(7.5)+6.11(11)}{17.33}=5.82 \mathrm{~m} \\
& y_{C T 1}=\frac{3.50(0)+2.09(3.5)+2.09(7.5)+4.20(11)}{11.89}=5.82 \mathrm{~m}
\end{aligned}
$$

Cálculos para la excentricidad de entrepiso. Coordenadas de los $C C$, ecuación (10)

$$
\begin{aligned}
& y_{C C 5}=\frac{23.77(3.75)}{23.77}=3.75 \mathrm{~m} \\
& y_{C C 4}=\frac{23.77(3.75)+25.75(5.50)}{49.52}=4.66 \mathrm{~m} \\
& y_{C C 3}=\frac{23.77(3.75)+25.75(5.50)+24.76(5.50)}{74.28}=4.94 \mathrm{~m} \\
& y_{C C 2}=\frac{23.77(3.75)+25.75(5.50)+24.76(5.50)+17.33(5.50)}{91.61}=5.05 \mathrm{~m} \\
& y_{C C 1}=\frac{23.77(3.75)+25.75(5.50)+24.76(5.50)+17.33(5.50)+11.89(6.30)}{103.50}=5.19 \mathrm{~m}
\end{aligned}
$$

Coordenadas de $C R$, ecuación (11)

$$
\begin{aligned}
& y_{C R 5}=\frac{6.48(0)+4.32(3.5)+12.97(7.5)}{23.77}=4.73 \mathrm{~m} \\
& y_{C R 4}=\frac{13.51(0)+9.0(3.5)+9.0(7.5)+18.01(11)}{49.52}=6.0 \mathrm{~m} \\
& y_{C R 3}=\frac{21.85(0)+13.11(3.5)+13.11(7.5)+26.22(11)}{74.29}=5.82 \mathrm{~m} \\
& y_{C R 2}=\frac{26.94(0)+16.17(3.5)+16.17(7.5)+32.33(11)}{91.61}=5.82 \mathrm{~m} \\
& y_{C R 1}=\frac{30.44(0)+18.26(3.5)+18.26(7.5)+36.53(11)}{103.50}=5.82 \mathrm{~m}
\end{aligned}
$$




\section{APÉNDICE B. CÁLCULO DE LOS CORTANTES TOTALES EN LOS ELEMENTOS RESISTENTES DEL ENTREPISO 5 DEL EDIFICIO ESTUDIADO}

Utilizando el PSD propuesto, se presenta el cálculo de los cortantes totales en los elementos resistentes del entrepiso 5 del edificio estudiado por Bazán y Meli (1998). Para la dirección $X$ :

$$
\rho=\frac{1}{7.5} \sqrt{\frac{621693}{4400}}=1.585 ; \quad \rho^{2}=2.5119 ; e=/ 0.98 / 7.5 /=0.13
$$

Para los dos elementos flexibles, marcos $1 \mathrm{X}$ y $2 \mathrm{X}$, el cálculo de los FAT y del cortante total se lleva a cabo con las ecuaciones (32) y (33), esto es

FATf $_{1 x}=1+\frac{0.631}{2.5119}(0.1+1.5(0.13))=1.074 ;$ por lo tanto: $V_{1 X}=1.074(6.48)=6.96 t$

FATf $_{2 x}=1+\frac{0.164}{2.5119}(0.1+1.5(0.13))=1.019$; por lo tanto: $V_{2 X}=1.019(4.32)=4.40 t$

Para el elemento rígido, marco 3X, se utilizan las ecuaciones (34) y (35), así se obtiene

$F A T r_{3 x}=1+\frac{0.369}{2.5119}(0.1-0.13)=0.996$; por lo tanto: $V_{3 X}=0.996(12.97)=12.91 t$

Para la dirección $Y$ :

$$
\begin{aligned}
& x_{C R 5}=\frac{26.45(0)+(1.43) 6.5+(19.66) 13.5}{47.54}=5.78 \mathrm{~m} \\
& \rho=\frac{1}{13.5} \sqrt{\frac{621693}{13300}=0.506 ; \rho^{2}=0.2565 ; e=/ 0.97 / 13.5 /=0.0719}
\end{aligned}
$$

Para los dos elementos flexibles, marcos $2 \mathrm{Y}$ y $3 \mathrm{Y}$, se tiene que

FATf $_{2 Y}=1+\frac{0.053}{0.2565}\left(0.1+1.5(0.0719)=1.043\right.$; por lo tanto: $V_{2 Y}=1.043(1.43)=1.49 t$

FATf $_{3 Y}=1+\frac{0.572}{0.2565}(0.1+1.5(0.0719))=1.464 ;$ por lo tanto: $V_{3 Y}=1.464(19.66)=28.78 t$

Para el elemento rígido, marco 1Y se obtiene

$F_{1 Y}=1+\frac{0.428}{0.256}(0.1-0.0719)=1.047$; por lo tanto: $V_{1 Y}=1.047(26.45)=27.69 t$ 